\title{
DYNAMICS AND ASYMPTOTIC PROFILES OF ENDEMIC EQUILIBRIUM FOR TWO FREQUENCY-DEPENDENT SIS EPIDEMIC MODELS WITH CROSS-DIFFUSION*
}

\author{
HUICONG LI, RUI PENG, AND TIAN XIANG ${ }^{\dagger}$
}

\begin{abstract}
This paper is concerned with two frequency-dependent SIS epidemic reaction-diffusion models in heterogeneous environment, with a cross-diffusion term modeling the effect that susceptible individuals tend to move away from higher concentration of infected individuals. It is first shown that the corresponding Neumann initial-boundary value problem in an $n$-dimensional bounded smooth domain possesses a unique global classical solution which is uniformly-in-time bounded regardless of the strength of the cross-diffusion and the spatial dimension $n$. It is further shown that, even in the presence of cross-diffusion, the models still admit threshold-type dynamics in terms of the basic reproduction number $\mathcal{R}_{0}$; that is, the unique disease free equilibrium is globally stable if $\mathcal{R}_{0}<1$, while if $\mathcal{R}_{0}>1$, the disease is uniformly persistent and there is an endemic equilibrium, which is globally stable in some special cases with weak chemotactic sensitivity. Our results on the asymptotic profiles of endemic equilibrium illustrate that restricting the motility of susceptible population may eliminate the infectious disease entirely for the first model with constant total population but fails for the second model with varying total population. In particular, this implies that such crossdiffusion does not contribute to the elimination of the infectious disease modelled by the second one.
\end{abstract}

\section{INTRODUCTION}

In this paper, we are interested in the following two diffusive SIS epidemic models with cross-diffusion and frequency-dependence:

$$
\begin{cases}S_{t}=d_{S} \Delta S+\chi \nabla \cdot(S \nabla I)-\beta(x) \frac{S I}{S+I}+\gamma(x) I, & x \in \Omega, t>0, \\ I_{t}=d_{I} \Delta I+\beta(x) \frac{S I}{S+I}-\gamma(x) I, & x \in \Omega, t>0, \\ \frac{\partial S}{\partial \nu}=\frac{\partial I}{\partial \nu}=0, & x \in \partial \Omega, t>0, \\ (S(x, 0), I(x, 0))=\left(S_{0}(x), I_{0}(x)\right), & x \in \Omega\end{cases}
$$

2010 Mathematics Subject Classification. 35K57; 35A01; 35B40; 35Q92; 92D25.

Key words and phrases. SIS epidemic reaction-diffusion model; Cross-diffusion; Global existence and boundedness; Endemic equilibrium; Persistence/extinction; Asymptotic profile.

${ }^{\dagger}$ Corresponding author.

${ }^{*} \mathrm{H}$. Li was partially supported by China Postdoctoral Science Foundation (No. 2016M590335) and NSF of China (Nos. 11701180, 11671143 and 11671144), R. Peng was partially supported by NSF of China (Nos. 11671175 and 11571200), the Priority Academic Program Development of Jiangsu Higher Education Institutions, Top-notch Academic Programs Project of Jiangsu Higher Education Institutions (No. PPZY2015A013) and Qing Lan Project of Jiangsu Province, and T. Xiang was partially supported by NSF of China (Nos. 11601516 and 11571363) and the Research Funds of Renmin University of China (No. 2018030199). 
and

$$
\begin{cases}S_{t}=d_{S} \Delta S+\chi \nabla \cdot(S \nabla I)+\Lambda(x)-S-\beta(x) \frac{S I}{S+I}+\gamma(x) I, & x \in \Omega, t>0 \\ I_{t}=d_{I} \Delta I+\beta(x) \frac{S I}{S+I}-\gamma(x) I, & x \in \Omega, t>0 \\ \frac{\partial S}{\partial \nu}=\frac{\partial I}{\partial \nu}=0, & x \in \partial \Omega, t>0 \\ (S(x, 0), I(x, 0))=\left(S_{0}(x), I_{0}(x)\right), & x \in \Omega .\end{cases}
$$

Here, $\Omega \subset \mathbb{R}^{n}(n \geq 1)$ is a bounded domain with smooth boundary $\partial \Omega$. The unknown functions $S(x, t)$ and $I(x, t)$, respectively, denote the population density of susceptible and infected individuals at location $x$ and time $t ; d_{S}$ and $d_{I}$ are positive constants measuring the random mobility of susceptible and infected populations respectively; the cross-diffusion term $\chi \nabla \cdot(S \nabla I)$ stands for the "chemotaxis" effect that susceptible individuals are "smart" and they tend to move away from higher concentration of infected individuals with the positive constant $\chi$ representing the magnitude of this effect; and $\beta(x)$ and $\gamma(x)$ are positive Hölder continuous functions on $\bar{\Omega}$ accounting for the rates of disease transmission and recovery at location $x$, respectively. In (1.2), the $S$-equation indicates that the susceptible population is subject to linear source $\Lambda-S$ with $\Lambda$ being a positive Hölder continuous function. The homogeneous Neumann boundary conditions mean there is no population flux crossing the boundary $\partial \Omega$. As for the initial data $\left(S_{0}, I_{0}\right)$, we assume throughout this paper that

$$
0 \leq S_{0} \in C(\bar{\Omega}), \quad I_{0} \in W^{1, \infty}(\Omega) \text { and } I_{0} \geq 0, \not \equiv 0 .
$$

Let

$$
N:=\int_{\Omega}\left(S_{0}(x)+I_{0}(x)\right) d x>0
$$

be the total number of individuals in $\Omega$ at the initial time $t=0$. By integrating both equations in (1.1) and then adding the resulting identities, one can easily see that the total population is conserved. That is,

$$
\int_{\Omega}(S(x, t)+I(x, t)) d x=N, \quad \forall t>0 .
$$

Throughout the text, we assume that $N$ is a given positive constant. Obviously, such conservation property no longer holds for system (1.2).

To investigate the effects of environmental heterogeneity and individual motility, Allen et al. [3] proposed the following frequency-dependent SIS (susceptibleinfected-susceptible) epidemic reaction-diffusion system.

$$
\begin{cases}\frac{\partial S}{\partial t}-d_{S} \Delta S=-\beta(x) \frac{S I}{S+I}+\gamma(x) I, & x \in \Omega, t>0, \\ \frac{\partial I}{\partial t}-d_{I} \Delta I=\beta(x) \frac{S I}{S+I}-\gamma(x) I, & x \in \Omega, t>0, \\ \frac{\partial S}{\partial \nu}=\frac{\partial I}{\partial \nu}=0, & x \in \partial \Omega, t>0, \\ S(x, 0)=S_{0}(x) \geq 0, I(x, 0)=I_{0}(x) \geq, \neq 0, & x \in \Omega .\end{cases}
$$

In [3], the authors defined the basic reproduction number $\mathcal{R}_{0}$ via a variational characterization and it was shown that the unique disease-free equilibrium (DFE) is globally asymptotically stable if $\mathcal{R}_{0}<1$, whereas there exists a unique endemic 
equilibrium (EE) if $\mathcal{R}_{0}>1$. Here, a $\operatorname{DFE}(S, I)$ is an equilibrium with $I \equiv 0$, whereas an $\operatorname{EE}(S, I)$ is a steady state with $I(x)>0$ for some $x \in \Omega$. The authors were particularly interested in the asymptotic behavior of the unique EE as $d_{S}$ approaches zero. Among other things, their results imply that, if the spatial environment can be modified to include low-risk sites and the movement of susceptible individuals can be restricted, then it may be possible to eliminate the infectious disease.

Although the existence and uniqueness of EE is proved in [3] when $\mathcal{R}_{0}>1$, the global stability of it was left open. In some special cases, the authors of [29] confirmed that it is indeed globally asymptotically stable. Further results concerning the asymptotic behavior of the EE of (1.5) were obtained by [28, 30]. On the other hand, with $\beta$ and $\gamma$ being functions of spatiotemporal variables and temporally periodic, the model (1.5) was treated by the second author and Zhao [31, and the theoretical findings of [31] imply that the combination of spatial heterogeneity and temporal periodicity can enhance the persistence of the disease. We refer interested readers to [2, 8, 9, 10, 11, 12, 15, 16, 18, 22, 24, 41] and the references therein for related research work on (1.5).

The model (1.2) with $\chi=0$ was studied by Li et al. 24], where comprehensive qualitative analysis has been performed and the findings indicate that a varying total population can enhance persistence of infectious disease, and hence the disease becomes more threatening and harder to control.

Biologically, the cross-diffusion introduced to the systems (1.1) and (1.2) represents a strategy that the susceptible implements to avoid infection by staying away from the infected (known as the repulsive chemotaxis phenomenon [7, 25, 33, 34]). The main purpose of this paper is to investigate the influence of such directed movement strategy of the susceptible population on the persistence or extinction of infectious diseases in the environment of spatial heterogeneity and random population movement via performing qualitative analysis on the systems (1.1) and (1.2). The cross-diffusion term $\chi \nabla \cdot(S \nabla I)$ has been widely shown to have a strong effect in driving solutions of the underlying models to blow up in finite/infinite time, as can be seen in the extensively studied Keller-Segel chemotaxis related systems [4, 20, 38, 39]. Thus, the global solvability of systems (1.1) and (1.2) needs to be seriously treated before we study their other dynamical properties. By a close inspection of the $I$-equation in (1.1) or (1.2), we find that the essential linearity not only enables us to obtain the $L^{\infty}$-bound of $I$, but also that of $\nabla I$, while this information is usually unavailable in most Keller-Segel models. With such a key observation, we are then able to establish the global existence and boundedness of classical solutions to (1.1) and (1.2) for arbitrary $\chi>0$ in any spatial dimensions; see Theorem 2.3. This result shows the cross-diffusion does not destroy the global solvability of the corresponding system without cross-diffusion.

As in [3], for our systems (1.1) and (1.2), we use the same definition of the basic reproduction number $\mathcal{R}_{0}$ since it determines the local stability of the unique DFE. Then we are also able to establish the threshold type dynamics in terms of $\mathcal{R}_{0}$. More specifically, we show that the unique DFE is in fact globally stable if $\mathcal{R}_{0}<1$ (see Theorems 3.5 and 6.1), which yield the extinction of infectious disease in the long run. While in the case of $\mathcal{R}_{0}>1$, a unique EE exists for system (1.1) whereas its uniqueness is unclear for system (1.2) since we are no longer able to reduce the equilibrium problem to a single equation due to the non-conservation of total population. In the special case that the transmission rate is proportional to the 
recovery rate throughout the habitat, it is proved that the unique homogeneous EE is globally stable when $\mathcal{R}_{0}>1$, provided that $\chi>0$ is suitably small; see Theorems [3.6 and 6.2, which cover and extend [29, Theorem 1.2] with $\chi=0$. Compared to the no cross-diffusion system (1.5), our results suggest that such directed movement strategy adopted by the susceptible with insignificant magnitude does not help to eliminate the infectious disease.

To study the effect of random motility of susceptible populations, we discuss the asymptotic behavior of the EE as $d_{S} \rightarrow 0$. For system (1.1) with constant total population, whenever $\mathcal{R}_{0}>1$ and the domain includes points where the transmission rate is smaller than the recovery rate, it is shown that the unique EE tends to a spatially inhomogeneous DFE as $d_{S} \rightarrow 0$. Furthermore, the density of the susceptible population of this limiting DFE, positive on low-risk sites (where the transmission rate is less than the recovery rate, i.e., where $\beta(x)<\gamma(x)$ ), must also be positive at some (but not all) high-risk sites (where the transmission rate is larger than the recovery rate, i.e., where $\beta(x)>\gamma(x))$. This result agrees with that of [3] for model (1.5) without directed diffusion. From the biological point of view, this in particular means that it is possible to eliminate the disease entirely in the habitat by restricting the random motility of susceptible individuals to be small. In stark contrast, for model (1.2) with varying total population, although we are not able to fully determine the asymptotic profile of EE for small $d_{S}>0$, Theorem 6.4 below implies that the disease still exists on the whole habitat for any given $\chi>0$, and therefore the introduction of cross-diffusion for the susceptible can not help to eliminate the disease. As a consequence, the theoretical finding in the current paper, in combination with the result of [24], suggests that the restriction of the diffusion rate of the susceptible is no longer an appropriate strategy for the eradication of infectious disease modelled by (1.2) where the total population number can vary.

The plan of this paper is organized as follows. In Section 2, we discuss the global existence and boundedness of solutions to models (1.1) and (1.2) based on a semigroup type argument. Section 3 is devoted to the threshold dynamics where the global stability of DFE and EE (in a special case) is studied. In Section 4, by reducing the equilibrium problem of (1.1) to a single equation, we establish the existence and uniqueness of EE. Asymptotic profile of the EE for small $d_{S}$ is then discussed in Section 5. Finally, in Section 6, we briefly investigate system (1.2) and point out the main differences.

\section{Global Existence and Boundedness}

In this section, we shall establish the global existence and boundedness property of classical solutions to (1.1) and (1.2) via semigroup theory. For the sake of reference, we present some known smoothing $L^{p}-L^{q}$ type estimates on the Neumann heat semigroup $\left(e^{t k \Delta}\right)_{t>0}$ on a bounded and smooth domain $\Omega$. One can find them in [36, Lemma 1.3], [6, Lemma 2.1] or [21, Lemma 2.1].

Lemma 2.1. For $k>0$, let $\left(e^{t k \Delta}\right)_{t>0}$ be the Neumann heat semigroup and $\lambda_{1}=$ : $\lambda_{1}(k)>0$ be the first positive Neumann eigenvalue of $-k \Delta$ on $\Omega$. Then there exist some positive constants $c_{i}(i=1,2,3,4)$ depending only on $k$ and $\Omega$ fulfilling

(i) If $1 \leq q \leq p \leq \infty$, then

$$
\left\|e^{t k \Delta} f\right\|_{L^{p}(\Omega)} \leq c_{1}\left(1+t^{-\frac{n}{2}\left(\frac{1}{q}-\frac{1}{p}\right)}\right)\|f\|_{L^{q}(\Omega)}, \quad \forall t>0
$$


holds for all $f \in L^{q}(\Omega)$.

(ii) If $1 \leq q \leq p \leq \infty$, then

$$
\left\|\nabla e^{t k \Delta} f\right\|_{L^{p}(\Omega)} \leq c_{2}\left(1+t^{-\frac{1}{2}-\frac{n}{2}\left(\frac{1}{q}-\frac{1}{p}\right)}\right) e^{-\lambda_{1} t}\|f\|_{L^{q}(\Omega)}, \quad \forall t>0
$$

holds for all $f \in L^{q}(\Omega)$.

(iii) If $2 \leq q \leq p<\infty$, then

$$
\left\|\nabla e^{t k \Delta} f\right\|_{L^{p}(\Omega)} \leq c_{3}\left(1+t^{-\frac{n}{2}\left(\frac{1}{q}-\frac{1}{p}\right)}\right) e^{-\lambda_{1} t}\|\nabla f\|_{L^{q}(\Omega)}, \quad \forall t>0
$$

holds for all $f \in W^{1, q}(\Omega)$.

(iv) If $1<q \leq p \leq \infty$, then

$$
\left\|e^{t k \Delta} \nabla \cdot f\right\|_{L^{p}(\Omega)} \leq c_{4}\left(1+t^{-\frac{1}{2}-\frac{n}{2}\left(\frac{1}{q}-\frac{1}{p}\right)}\right) e^{-\lambda_{1} t}\|f\|_{L^{q}(\Omega)}, \quad \forall t>0
$$

holds for all $f \in\left(L^{q}(\Omega)\right)^{n}$.

For notational convenience, throughout the paper, we shall denote

$$
m^{*}=\max _{x \in \bar{\Omega}} m(x) \quad \text { and } \quad m_{*}=\min _{x \in \bar{\Omega}} m(x)
$$

with $m \in\{\beta, \gamma\}$.

Using Lemma 2.1 and Banach's contraction mapping theorem, one can establish the local solvability of systems (1.1) and (1.2) . For details of the similar reasoning, we refer to [17, Theorem 3.1] and [37, Lemma 1.1]; see also [4, Lemma 3.1].

Lemma 2.2. Assume that the initial data fulfills (1.3). Then there exists $T_{\max } \in$ $(0, \infty]$ and a uniquely determined pair of nonnegative functions

$$
\begin{aligned}
& S \in C\left(\bar{\Omega} \times\left[0, T_{\max }\right)\right) \cap C^{2,1}\left(\bar{\Omega} \times\left(0, T_{\max }\right)\right) \\
& I \in C\left(\bar{\Omega} \times\left[0, T_{\max }\right)\right) \cap C^{2,1}\left(\bar{\Omega} \times\left(0, T_{\max }\right)\right) \cap L_{\mathrm{loc}}^{\infty}\left(\left[0, T_{\max }\right) ; W^{1, p}(\Omega)\right)
\end{aligned}
$$

with any $p>1$ such that $(S, I)$ solves (1.1) classically in $\Omega \times\left(0, T_{\max }\right)$. Furthermore, if $T_{\max }<\infty$, then, for any $p>1$,

$$
\|S(\cdot, t)\|_{L^{\infty}(\Omega)}+\|I(\cdot, t)\|_{W^{1, p}(\Omega)} \rightarrow \infty \text { as } t \nearrow T_{\max } .
$$

The same local-in-time well-posedness holds true for model (1.2). For model (1.1), the conservation law (1.4) holds in $\left(0, T_{\max }\right)$; for model (1.2), the following uniform $L^{1}$-estimate for $S+I$ holds in $\left(0, T_{\max }\right)$ :

$$
\begin{aligned}
& \int_{\Omega}\left(S(x, t)+\left(1+\frac{1}{2 \beta^{*}}\right) I(x, t)\right) d x \\
& \leq \int_{\Omega}\left(S_{0}(x)+\left(1+\frac{1}{2 \beta^{*}}\right) I_{0}(x)\right) d x+\frac{2 \int_{\Omega} \Lambda(x) d x}{\min \left\{1, \frac{2 \gamma_{*}}{1+2 \beta^{*}}\right\}}=: \hat{N}, \quad \forall t>0 .
\end{aligned}
$$

Proof. As noted above, the statements concerning the local-in-time existence of classical solutions to the initial-boundary value problems (1.1) and (1.2) and the criterion (2.1) are well-studied. The nonnegativity (positivity) of $(S, I)$ follows simply from the maximum principle. Due to no flux boundary conditions, upon integration of the $S$ - and $I$-equation in (1.1), the conservation law (1.4) follows trivially. For 
the uniform $L^{1}$-bound in (2.2), by straightforward computations, we deduce from the IBVP (1.2) that

$$
\begin{aligned}
& \frac{d}{d t} \int_{\Omega}\left(S(x, t)+\left(1+\frac{1}{2 \beta^{*}}\right) I(x, t)\right) d x \\
& =\int_{\Omega} \Lambda(x) d x-\int_{\Omega} S(x, t) d x+\frac{1}{2 \beta^{*}} \int_{\Omega} \frac{\beta(x) S(x, t) I(x, t)}{S(x, t)+I(x, t)} d x-\frac{1}{2 \beta^{*}} \int_{\Omega} \gamma(x) I(x, t) d x \\
& \leq \int_{\Omega} \Lambda(x) d x-\frac{1}{2} \int_{\Omega} S(x, t) d x-\frac{\gamma_{*}}{2 \beta^{*}} \int_{\Omega} I(x, t) d x \\
& \leq \int_{\Omega} \Lambda(x) d x-\frac{1}{2} \min \left\{1, \frac{2 \gamma_{*}}{1+2 \beta^{*}}\right\} \int_{\Omega}\left(S(x, t)+\left(1+\frac{1}{2 \beta^{*}}\right) I(x, t)\right) d x .
\end{aligned}
$$

Solving this standard Gronwall differential inequality, we arrive at (2.2).

Our main result on global existence and uniform-in-time boundedness for (1.1) and (1.2) reads precisely as follows.

Theorem 2.3. Assume that the initial data fulfills (1.3). Then each of the crossdiffusive SIS models (1.1) and (1.2) possesses a uniquely determined global classical solution $(S, I)$ for which both $S$ and $I$ are positive and bounded in $\bar{\Omega} \times(0, \infty)$. That is, there exists some $M>0$ depending on initial data and the model parameters such that

$$
\|S(\cdot, t)\|_{L^{\infty}(\Omega)}+\|I(\cdot, t)\|_{W^{1, \infty}(\Omega)} \leq M, \quad \forall t>0 .
$$

Moreover, there exists some $M^{\prime}>0$ independent of initial data fulfilling

$$
\|S(\cdot, t)\|_{L^{\infty}(\Omega)}+\|I(\cdot, t)\|_{L^{\infty}(\Omega)} \leq M^{\prime}, \quad \forall t>T
$$

for some large $T>0$. Furthermore, the $L^{\infty}$-bound of $I$ is uniform in $\chi$, i.e.,

$$
\|I(\cdot, t)\|_{L^{\infty}(\Omega)} \leq M_{I}\left(n, \Omega, \gamma_{*}, \beta^{*}\right)\left(1+\frac{1}{d_{I}}\right)^{n} \max \left\{\left\|I_{0}\right\|_{L^{\infty}(\Omega)}, \tilde{N}\right\}, \quad \forall t>0 .
$$

Here, $\tilde{N}=N$ for model (1.1) and $\tilde{N}=\hat{N}$ with $\hat{N}$ defined by (2.2) for model (1.2).

Proof. Thanks to Lemma 2.2, we shall first show $T_{\max }=\infty$ and then the global boundedness of $(S, I)$. To this end, we start with the $I$-associated problem

$$
\begin{cases}I_{t}=d_{I} \Delta I+B(x, t) I, & x \in \Omega, t>0, \\ \frac{\partial I}{\partial \nu}=0, & x \in \partial \Omega, t>0, \\ I(x, 0)=I_{0}(x), & x \in \Omega,\end{cases}
$$

where

$$
B(x, t)=\beta(x) \frac{S(x, t)}{S(x, t)+I(x, t)}-\gamma(x) .
$$

It is clear that $B$ is uniformly bounded by $\left(\beta^{*}+\gamma^{*}\right)$ and is locally Lipschitz on $\Omega \times\left(0, T_{\max }\right)$. Furthermore, $\|I(\cdot, t)\|_{L^{1}(\Omega)} \leq \tilde{N}$ for $t \in\left(0, T_{\max }\right)$ due to the validity of (1.4) and (2.2) in $\left(0, T_{\max }\right)$. Thus, [1, Theorem 3.1 on " $L^{1}$-boundedness implies $L^{\infty}$-boundedness"] (see also [31, Lemma 3.1]) applied to (2.6) yields the existence of a positive constant $C_{1}$ such that

$$
\|I(\cdot, t)\|_{L^{\infty}(\Omega)} \leq C_{1}\left(d_{I}, \Omega, n, \beta, \gamma\right), \quad \forall t \in\left(0, T_{\max }\right) .
$$

Thanks to the $L^{1}$-bound in (1.4) and (2.2), the bound for $I$ in (2.5) indeed could be obtained via standard Moser iteration applied to (2.6). 
Next, according to the variation-of-constants formula, we have

$$
I(\cdot, t)=e^{t d_{I} \Delta} I_{0}+\int_{0}^{t} e^{(t-\tau) d_{I} \Delta}\left(\frac{\beta S I}{S+I}-\gamma I\right)(\cdot, \tau) d \tau, \quad \forall t \in\left(0, T_{\max }\right),
$$

from which it follows that

$$
\begin{aligned}
\|\nabla I(\cdot, t)\|_{L^{\infty}(\Omega)} \leq & \left\|\nabla e^{t d_{I} \Delta} I_{0}\right\|_{L^{\infty}(\Omega)} \\
& +\int_{0}^{t}\left\|\nabla e^{(t-\tau) d_{I} \Delta}\left(\frac{\beta S I}{S+I}-\gamma I\right)(\cdot, \tau)\right\|_{L^{\infty}(\Omega)} d \tau .
\end{aligned}
$$

For $p \in[2, \infty)$, Lemma 2.1 (iii) entails, for all $t>0$,

$$
\left\|\nabla e^{t d_{I} \Delta} I_{0}\right\|_{L^{p}(\Omega)} \leq 2 c_{3} e^{-\lambda_{1}\left(d_{I}\right) t}\left\|\nabla I_{0}\right\|_{L^{p}(\Omega)} \leq 2 c_{3} e^{-\lambda_{1}\left(d_{I}\right) t} \max \left\{|\Omega|^{\frac{1}{2}}, 1\right\}\left\|\nabla I_{0}\right\|_{L^{\infty}(\Omega)} .
$$

This immediately implies the existence of a constant $C_{2}>0$ such that

$$
\left\|\nabla e^{t d_{I} \Delta} I_{0}\right\|_{L^{\infty}(\Omega)} \leq C_{2}\left\|I_{0}\right\|_{W^{1, \infty}(\Omega)}, \quad \forall t>0 .
$$

On the other hand, it follows from Lemma 2.1 (ii) that

$$
\begin{aligned}
& \left\|\nabla e^{(t-\tau) d_{I} \Delta}\left(\frac{\beta S I}{S+I}-\gamma I\right)(\cdot, \tau)\right\|_{L^{\infty}(\Omega)} \\
& \leq c_{2}\left(1+(t-\tau)^{-\frac{1}{2}}\right) e^{-\lambda_{1}\left(d_{I}\right)(t-\tau)}\left(\beta^{*}+\gamma^{*}\right)\|I(\cdot, \tau)\|_{L^{\infty}(\Omega)}, \quad \forall t \in\left(\tau, T_{\max }\right) .
\end{aligned}
$$

This along with (2.8), (2.9) and the $L^{\infty}$-boundedness of $I$ yields that

$$
\begin{aligned}
\|\nabla I(\cdot, t)\|_{L^{\infty}(\Omega)} & \leq C_{2}\left\|I_{0}\right\|_{W^{1, \infty}(\Omega)}+C_{3} \int_{0}^{t}\left(1+(t-\tau)^{-\frac{1}{2}}\right) e^{-\lambda_{1}\left(d_{I}\right)(t-\tau)} d \tau \\
& \leq C_{4}, \quad \forall t \in\left(0, T_{\max }\right) .
\end{aligned}
$$

Now, we are ready to derive the $L^{\infty}$-bound of $S$. For definiteness, we will first work on (1.1) and just give a quick remark for (1.2) in the end. Fix any $0<T<T_{\max }$ and $p>n$. We rewrite the $S$-equation in (1.1) as

$$
S_{t}-d_{S} \Delta S+S=\chi \nabla \cdot(S \nabla I)+S-\beta \frac{S}{S+I} I+\gamma I,
$$

which gives, upon an application of the variation-of-constants formula,

$$
\begin{aligned}
S(\cdot, t)= & e^{t\left(d_{S} \Delta-1\right)} S_{0}+\chi \int_{0}^{t} e^{(t-\tau)\left(d_{S} \Delta-1\right)} \nabla \cdot(S(\cdot, \tau) \nabla I(\cdot, \tau)) d \tau \\
& +\int_{0}^{t} e^{(t-\tau)\left(d_{S} \Delta-1\right)}\left(S-\beta \frac{S}{S+I} I+\gamma I\right)(\cdot, \tau) d \tau, \quad \forall t \in(0, T) .
\end{aligned}
$$

Taking supremum on both sides, we obtain

$$
\begin{aligned}
\|S(\cdot, t)\|_{L^{\infty}(\Omega)} \leq & \left\|e^{t\left(d_{S} \Delta-1\right)} S_{0}\right\|_{L^{\infty}(\Omega)} \\
& +\chi \int_{0}^{t}\left\|e^{(t-\tau)\left(d_{S} \Delta-1\right)} \nabla \cdot(S(\cdot, \tau) \nabla I(\cdot, \tau))\right\|_{L^{\infty}(\Omega)} d \tau \\
& +\int_{0}^{t}\left\|e^{(t-\tau)\left(d_{S} \Delta-1\right)}\left(S-\beta \frac{S}{S+I} I+\gamma I\right)(\cdot, \tau)\right\|_{L^{\infty}(\Omega)} d \tau \\
= & \text { I }+ \text { II }+ \text { III, } \quad \forall t \in(0, T) .
\end{aligned}
$$

We first deduce from the maximum principle that

$$
\mathrm{I}=e^{-t}\left\|e^{t d_{S} \Delta} S_{0}\right\|_{L^{\infty}(\Omega)} \leq e^{-t}\left\|S_{0}\right\|_{L^{\infty}(\Omega)}, \quad \forall t \in(0, T) .
$$


For convenience, we define

$$
R=R(T):=\sup _{t \in(0, T)}\|S(\cdot, t)\|_{L^{\infty}(\Omega)} .
$$

Using Lemma 2.1 (iv) and (2.10), we are led to

$$
\begin{aligned}
\mathrm{II} & \leq \chi \int_{0}^{t} e^{-(t-\tau)}\left\|e^{(t-\tau) d_{S} \Delta} \nabla \cdot(S(\cdot, \tau) \nabla I(\cdot, \tau))\right\|_{L^{\infty}(\Omega)} d \tau \\
& \leq C_{5} \int_{0}^{t} e^{-(t-\tau)}\left(1+(t-\tau)^{-\frac{1}{2}-\frac{n}{2 p}}\right)\|S(\cdot, \tau) \nabla I(\cdot, \tau)\|_{L^{p}(\Omega)} d \tau \\
& \leq C_{6} \int_{0}^{t} e^{-(t-\tau)}\left(1+(t-\tau)^{-\frac{1}{2}-\frac{n}{2 p}}\right)\|S(\cdot, \tau)\|_{L^{p}(\Omega)} d \tau \\
& \leq C_{6} \int_{0}^{t} e^{-(t-\tau)}\left(1+(t-\tau)^{-\frac{1}{2}-\frac{n}{2 p}}\right)\|S(\cdot, \tau)\|_{L^{\infty}(\Omega)}^{1-\frac{1}{p}}\|S(\cdot, \tau)\|_{L^{1}(\Omega)}^{\frac{1}{p}} d \tau \\
& \leq C_{6} R^{1-\frac{1}{p}} N^{\frac{1}{p}} \int_{0}^{t} e^{-(t-\tau)}\left(1+(t-\tau)^{-\frac{1}{2}-\frac{n}{2 p}}\right) d \tau \\
& \leq C_{6} C_{7} R^{1-\frac{1}{p}} N^{\frac{1}{p}}, \quad \forall t \in(0, T),
\end{aligned}
$$

where the fact that $p>n$ was used to guarantee

$$
\begin{aligned}
C_{7} & =\int_{0}^{t} e^{-(t-\tau)}\left(1+(t-\tau)^{-\frac{1}{2}-\frac{n}{2 p}}\right) d \tau \\
& \leq 1+\int_{0}^{\infty} e^{-\sigma} \sigma^{-\frac{1}{2}-\frac{n}{2 p}} d \sigma=1+\Gamma\left(\frac{1}{2}-\frac{n}{2 p}\right)<\infty .
\end{aligned}
$$

To estimate III, using (1.4) and Lemma 2.1 (i), we deduce

$$
\begin{aligned}
\mathrm{III} \leq & \int_{0}^{t} e^{-(t-\tau)}\left\|e^{(t-\tau) d_{S} \Delta} S(\cdot, \tau)\right\|_{L^{\infty}(\Omega)} d \tau \\
& +\int_{0}^{t} e^{-(t-\tau)}\left\|e^{(t-\tau) d_{S} \Delta}\left(-\beta \frac{S}{S+I} I+\gamma I\right)(\cdot, \tau)\right\|_{L^{\infty}(\Omega)} d \tau \\
\leq & c_{1} \int_{0}^{t} e^{-(t-\tau)}\left(1+(t-\tau)^{-\frac{n}{2 p}}\right)\|S(\cdot, \tau)\|_{L^{p}(\Omega)} d \tau \\
& +\int_{0}^{t} e^{-(t-\tau)}\left(\beta^{*}+\gamma^{*}\right)\|I(\cdot, \tau)\|_{L^{\infty}(\Omega)} d \tau \\
\leq & c_{1} \int_{0}^{t} e^{-(t-\tau)}\left(1+(t-\tau)^{-\frac{n}{2 p}}\right)\|S(\cdot, \tau)\|_{L^{\infty}(\Omega)}^{1-\frac{1}{p}}\|S(\cdot, \tau)\|_{L^{1}(\Omega)}^{\frac{1}{p}} d \tau+C_{8} \\
\leq & c_{1} C_{9} R^{1-\frac{1}{p}} N^{\frac{1}{p}}+C_{8}
\end{aligned}
$$

with

$$
C_{9}=\int_{0}^{\infty} e^{-\sigma}\left(1+\sigma^{-\frac{n}{2 p}}\right) d \sigma<\infty
$$

Substituting (2.12), (2.13) and (2.14) into (2.11), we conclude that

$$
R \leq C_{10} R^{1-\frac{1}{p}}+C_{11},
$$


with $C_{10}=\left(C_{6} C_{7}+c_{1} C_{9}\right) N^{\frac{1}{p}}$ and $C_{11}=\left\|S_{0}\right\|_{L^{\infty}(\Omega)}+C_{8}$. Upon an elementary argument, we infer from the above inequality that

$$
R \leq \max \left\{\left(\frac{C_{11}}{C_{10}}\right)^{\frac{p}{p-1}}, \quad\left(2 C_{10}\right)^{p}\right\} .
$$

Since $T \in\left(0, T_{\max }\right)$ is arbitrary, we conclude there must exist $C>0$ such that

$$
\|S(\cdot, t)\|_{L^{\infty}(\Omega)} \leq C, \quad \forall t \in\left(0, T_{\max }\right) .
$$

This in conjunction with (2.7), (2.10) and (2.1) indicates that $T_{\max }=\infty$.

Now, we proceed to find an upper bound of $\|S(\cdot, t)+I(\cdot, t)\|_{L^{\infty}(\Omega)}$ that is independent of initial data for large $t$. In fact, a use of [31, Lemma 3.1] to problem (2.6) provides some constant $C_{12}>0$ independent of initial data and $T_{1}>0$ such that

$$
\|I(\cdot, t)\|_{L^{\infty}(\Omega)} \leq C_{12}, \quad t \geq T_{1} .
$$

Next, we represent $I$ in the following way:

$I(\cdot, t)=e^{\left(t-T_{1}\right)\left(d_{I} \Delta-1\right)} I\left(\cdot, T_{1}\right)+\int_{T_{1}}^{t} e^{(t-\tau)\left(d_{I} \Delta-1\right)}\left(I+\beta \frac{S I}{S+I}-\gamma I\right)(\cdot, \tau) d \tau, \quad \forall t>T_{1}$.

Then using a parallel argument to the one used to derive (2.10), it is easily seen there exists $T_{2}>T_{1}$, such that

$$
\|\nabla I(\cdot, t)\|_{L^{\infty}(\Omega)} \leq C_{13}, \quad \forall t \geq T_{2},
$$

for some constant $C_{13}>0$ independent of the initial data. Next representing $S$ as

$$
\begin{aligned}
S(\cdot, t)= & e^{\left(t-T_{2}\right)\left(d_{S} \Delta-1\right)} S\left(\cdot, T_{2}\right)+\chi \int_{T_{2}}^{t} e^{(t-\tau)\left(d_{S} \Delta-1\right)} \nabla \cdot(S(\cdot, \tau) \nabla I(\cdot, \tau)) d \tau \\
& +\int_{T_{2}}^{t} e^{(t-\tau)\left(d_{S} \Delta-1\right)}\left(S-\beta \frac{S}{S+I} I+\gamma I\right)(\cdot, \tau) d \tau, \quad \forall t>T_{2},
\end{aligned}
$$

and proceeding in the same fashion as we did to obtain (2.15), we can conclude that actually $\|S(\cdot, t)\|_{L^{\infty}(\Omega)}$ can be bounded by a positive constant independent of the initial data for $t$ sufficiently large. This proves the theorem for model (1.1).

Armed with the uniform $W^{1, \infty}$-bound for $I$ as obtained in (2.7) and (2.10) and the uniform $L^{1}$-bound for $S+I$ in (2.2), with minor modifications, we can repeat the proceeding arguments for (1.1) to derive the assertions of the theorem for (1.2).

\section{Threshold Dynamics in terms of $\mathcal{R}_{0}$}

In this section, we aim to study the global asymptotic stability of nonnegative steady states of (1.1) with the constraint (1.4). It is straightforward to see that the DFE exists uniquely and is given by

$$
(\tilde{S}, 0)=\left(\frac{N}{|\Omega|}, 0\right)
$$

For our model (1.1), as in [3], we define the basic reproduction number by

$$
\mathcal{R}_{0}=\sup _{0 \neq \varphi \in H^{1}(\Omega)} \frac{\int_{\Omega} \beta \varphi^{2} d x}{\int_{\Omega}\left(d_{I}|\nabla \varphi|^{2}+\gamma \varphi^{2}\right) d x} .
$$

Indeed, one can follow the idea of next generation operators in [31] to introduce the basic reproduction number, which coincides with $\mathcal{R}_{0}$. Observe that $\mathcal{R}_{0}$ is independent of the diffusion rate $d_{S}>0$. 
Proposition 3.1. The DFE is stable if $\mathcal{R}_{0}<1$, and it is unstable if $\mathcal{R}_{0}>1$.

The proof of Proposition 3.1] is the same as that of [3, Lemma 2.4] and so the details are omitted here. In addition, the following qualitative properties of $\mathcal{R}_{0}$ were also established in [3].

Proposition 3.2. The following assertions hold.

(a) $\mathcal{R}_{0}$ is a monotone decreasing function of $d_{I}$ with $\mathcal{R}_{0} \rightarrow \max \{\beta(x) / \gamma(x)$ : $x \in \bar{\Omega}\}$ as $d_{I} \rightarrow 0$ and $\mathcal{R}_{0} \rightarrow \int_{\Omega} \beta / \int_{\Omega} \gamma$ as $d_{I} \rightarrow \infty ;$

(b) if $\int_{\Omega} \beta(x) d x<\int_{\Omega} \gamma(x) d x$, then there exists a threshold value $d_{I}^{*} \in(0, \infty)$ such that $\mathcal{R}_{0}>1$ for $d_{I}<d_{I}^{*}$ and $\mathcal{R}_{0}<1$ for $d_{I}>d_{I}^{*}$;

(c) if $\int_{\Omega} \beta(x) d x \geq \int_{\Omega} \gamma(x) d x$, then $\mathcal{R}_{0}>1$ for all $d_{I}$.

Furthermore, it was shown in [3] that $1-\mathcal{R}_{0}$ has the same sign as $\lambda^{*}$ with $\lambda^{*}$ being the principal eigenvalue of the following eigenvalue problem.

$$
\begin{cases}-d_{I} \Delta \phi+(\gamma-\beta) \phi=\lambda \phi, & x \in \Omega \\ \frac{\partial \phi}{\partial \nu}=0, & x \in \partial \Omega\end{cases}
$$

Notice that the $I$-equation verifies

$$
I_{t}=d_{I} \Delta I+\beta \frac{S I}{S+I}-\gamma I \leq d_{I} \Delta I+(\beta-\gamma) I, \quad x \in \Omega, t>0 .
$$

Based on (3.2) and (3.3), we first have a simple observation, when $\mathcal{R}_{0}<1$, that $I(x, t)$ decays to 0 exponentially as $t \rightarrow \infty$. In fact, in such case, the principal eigenvalue $\lambda^{*}$ corresponding to (3.2) is positive. Let $\phi^{*}>0$ be the principal eigenfunction and let $M$ be a positive constant such that $I_{0}(x) \leq M \phi^{*}(x)$ for $x \in \Omega$. Then a direct application from (3.2) and (3.3) shows that $M e^{-\bar{\lambda}^{*} t} \phi^{*}(x)$ is a super-solution to the $I$-equation and hence the comparison principle yields that

$$
I(x, t) \leq M e^{-\lambda^{*} t} \phi^{*}(x), \quad x \in \Omega, t>0 .
$$

In the sequel, we shall employ this important information to derive the global asymptotic stability of DFE under the assumption that $\mathcal{R}_{0}<1$, and this is achieved through a chain of simple lemmas.

For convenience, we set $w(x, t)=S(x, t)+I(x, t)$ for $x \in \bar{\Omega}$ and $t \geq 0$. Then it can be readily checked from the IBVP (1.1) that $w$ satisfies

$$
\begin{cases}w_{t}=d_{S} \Delta w+\nabla \cdot\left(\left(d_{I}-d_{S}\right) \nabla I+\chi S \nabla I\right), & x \in \Omega, t>0, \\ \frac{\partial w}{\partial \nu}=0, & x \in \partial \Omega, t>0, \\ w(x, 0)=S_{0}(x)+I_{0}(x), & x \in \Omega .\end{cases}
$$

The following result serves as a key starting point towards our stabilization analysis for the $\operatorname{DFE}(\tilde{S}, 0)$.

Lemma 3.3. The ansatz $w$ satisfies the property that

$$
\int_{0}^{\infty} \int_{\Omega}|\nabla w|^{2} d x d t<\infty
$$

Proof. Multiplying the $I$-equation by $I$ and integrating over $\Omega$, we are led to

$$
\frac{1}{2} \frac{d}{d t} \int_{\Omega} I^{2} d x=-d_{I} \int_{\Omega}|\nabla I|^{2} d x+\int_{\Omega}\left(\beta \frac{S I}{S+I}-\gamma I\right) I d x .
$$


Thanks to (3.4), we have

$$
\begin{aligned}
d_{I} \int_{0}^{T} \int_{\Omega}|\nabla I|^{2} d x d t & =\frac{1}{2} \int_{\Omega} I_{0}^{2} d x-\frac{1}{2} \int_{\Omega} I^{2}(\cdot, T) d x+\int_{0}^{T} \int_{\Omega}\left(\beta \frac{S}{S+I}-\gamma\right) I^{2} d x d t \\
& \leq \frac{1}{2} \int_{\Omega} I_{0}^{2} d x+\beta^{*} \int_{0}^{T} \int_{\Omega}\left(M e^{-\lambda^{*} t} \phi^{*}(x)\right)^{2} d x d t \\
& \leq C_{1}, \quad \forall T>0,
\end{aligned}
$$

for some positive constant $C_{1}$ independent of $T>0$. Similarly, from the $S$-equation and the fact that $S$ and $I$ are bounded, we deduce

$$
\begin{aligned}
\frac{1}{2} \frac{d}{d t} \int_{\Omega} S^{2} d x= & -d_{S} \int_{\Omega}|\nabla S|^{2} d x-\chi \int_{\Omega} S \nabla I \cdot \nabla S d x-\int_{\Omega} \beta \frac{S^{2} I}{S+I} d x+\int_{\Omega} \gamma S I d x \\
\leq & -d_{S} \int_{\Omega}|\nabla S|^{2} d x+\frac{d_{S}}{2} \int_{\Omega}|\nabla S|^{2} d x+\frac{\chi^{2}}{2 d_{S}} \int_{\Omega} S^{2}|\nabla I|^{2} d x \\
& +\gamma^{*}\|S(\cdot, t)\|_{L^{\infty}(\Omega)} \int_{\Omega} I d x \\
\leq & -\frac{d_{S}}{2} \int_{\Omega}|\nabla S|^{2} d x+\frac{\chi^{2}}{2 d_{S}}\|S(\cdot, t)\|_{L^{\infty}(\Omega)}^{2} \int_{\Omega}|\nabla I|^{2} d x \\
& +\gamma^{*}\|S(\cdot, t)\|_{L^{\infty}(\Omega)} \int_{\Omega} I d x .
\end{aligned}
$$

As a result,

$$
\begin{aligned}
d_{S} \int_{0}^{T} \int_{\Omega}|\nabla S|^{2} d x d t & \leq \int_{\Omega} S_{0}^{2} d x+C_{2} \int_{0}^{T} \int_{\Omega}|\nabla I|^{2} d x d t+C_{2} \int_{0}^{T} \int_{\Omega} I d x d t \\
& \leq C_{3}, \quad \forall T>0,
\end{aligned}
$$

due to (3.7) and (3.4). Clearly, by the definition of $w$, the desired estimate (3.6) follows from (3.7) and (3.8).

With the aid of the decaying property (3.6), we next show that $w_{t}$ decays at least in the dual space of $H^{1}(\Omega)$ in the large time limit.

Lemma 3.4. The solution $w$ of (3.5) satisfies

$$
\int_{0}^{\infty}\left\|w_{t}(\cdot, t)\right\|_{\left(H^{1}(\Omega)\right)^{*}}^{2} d t<\infty
$$

Proof. For any test function $\varphi \in H^{1}(\Omega)$, we obtain from (3.5) that

$$
\begin{aligned}
\int_{\Omega} w_{t} \varphi d x= & -d_{S} \int_{\Omega} \nabla w \cdot \nabla \varphi d x-\int_{\Omega}\left[\left(d_{I}-d_{S}\right) \nabla I+\chi S \nabla I\right] \cdot \nabla \varphi d x \\
\leq & d_{S}\|\nabla w(\cdot, t)\|_{L^{2}(\Omega)}\|\nabla \varphi\|_{L^{2}(\Omega)} \\
& +\left[\left(d_{I}+d_{S}\right)\|\nabla I(\cdot, t)\|_{L^{2}(\Omega)}+\chi\|S(\cdot, t)\|_{L^{\infty}(\Omega)}\|\nabla I(\cdot, t)\|_{L^{2}(\Omega)}\right]\|\nabla \varphi\|_{L^{2}(\Omega)} \\
\leq & {\left[d_{S}\|\nabla w(\cdot, t)\|_{L^{2}(\Omega)}+\left(d_{I}+d_{S}+\chi\|S(\cdot, t)\|_{L^{\infty}(\Omega)}\right)\|\nabla I(\cdot, t)\|_{L^{2}(\Omega)}\right]\|\varphi\|_{H^{1}(\Omega)} . }
\end{aligned}
$$

This shows precisely that

$$
\left\|w_{t}(\cdot, t)\right\|_{\left(H^{1}(\Omega)\right)^{*}} \leq d_{S}\|\nabla w(\cdot, t)\|_{L^{2}(\Omega)}+\left(d_{I}+d_{S}+\chi\|S(\cdot, t)\|_{L^{\infty}(\Omega)}\right)\|\nabla I(\cdot, t)\|_{L^{2}(\Omega)} .
$$


As a consequence of Theorem 2.3, Lemma 3.3 and (3.7), we deduce

$$
\int_{0}^{\infty}\left\|w_{t}(\cdot, t)\right\|_{\left(H^{1}(\Omega)\right)^{*}}^{2} d t \leq C \int_{0}^{\infty}\|\nabla w(\cdot, t)\|_{L^{2}(\Omega)}^{2} d t+C \int_{0}^{\infty}\|\nabla I(\cdot, t)\|_{L^{2}(\Omega)}^{2} d t<\infty .
$$

This completes the proof of the lemma.

With the help of Lemmas 3.3 and 3.4, using a somewhat standard argument as in [35] and [40], we now can establish the global stability of DFE.

Theorem 3.5. If the basic reproduction number $\mathcal{R}_{0}<1$, then the unique globalin-time bounded and classical solution $(S, I)$ of (1.1) satisfies $(S, I) \rightarrow\left(\frac{N}{|\Omega|}, 0\right)$ in $C(\bar{\Omega})$ as $t \rightarrow \infty$.

Proof. We have already shown in (3.4) that $I(x, t) \rightarrow 0$ uniformly for $x \in \bar{\Omega}$ as $t \rightarrow \infty$. Recall that $w=S+I$, hence, it suffices to prove

$$
\left\|w(\cdot, t)-\frac{N}{|\Omega|}\right\|_{C(\bar{\Omega})} \rightarrow 0, \quad \text { as } t \rightarrow \infty .
$$

We shall show (3.9) by a contradiction argument. Suppose otherwise, then there exists a sequence $\left\{t_{k}\right\}_{k \in \mathbb{N}}$ with $t_{k} \rightarrow \infty$ as $k \rightarrow \infty$ such that

$$
\inf _{k \in \mathbb{N}}\left\|w\left(\cdot, t_{k}\right)-\frac{N}{|\Omega|}\right\|_{C(\bar{\Omega})}>0 \text {. }
$$

Since $\|S(\cdot, t)+I(\cdot, t)\|_{L^{\infty}(\Omega)}$ is bounded for $t>0$ by Theorem 2.3, the standard parabolic regularity for bounded solutions of parabolic equations (c.f. [23, 32] or [5, Theorem A2]) applied first to the $I$-equation in (1.1) tells us that $I(\cdot, t)$ is bounded in $C^{2}(\bar{\Omega})$ and then the Hölder regularity [5, Theorem A1] applied to (3.5) (see more detailed reasonings leading to (3.19) below) gives us that $\|S(\cdot, t)+I(\cdot, t)\|_{C^{\alpha}(\bar{\Omega})}=$ $\|w(\cdot, t)\|_{C^{\alpha}(\bar{\Omega})}$ is bounded for all $t \geq 2$ for some $\alpha \in(0,1)$. An application of the Arzelà-Ascoli Theorem yields that $\{w(\cdot, t)\}_{t \geq 2}$ is relatively compact in $C(\bar{\Omega})$. Thus, we can extract a subsequence, still denoted by $\left\{t_{k}\right\}_{k \in \mathbb{N}}$, such that

$$
w\left(\cdot, t_{k}\right) \rightarrow w_{\infty} \quad \text { in } C(\bar{\Omega}), \quad \text { as } k \rightarrow \infty
$$

for some $0 \leq w_{\infty} \in C(\bar{\Omega})$. First, the weak stabilization of $w_{t}$ in Lemma 3.4 entails

$$
\begin{aligned}
\int_{t_{k}}^{t_{k}+1}\left\|w(\cdot, t)-w\left(\cdot, t_{k}\right)\right\|_{\left(H^{1}(\Omega)\right)^{*}}^{2} d t & =\int_{t_{k}}^{t_{k}+1}\left\|\int_{t_{k}}^{t} w_{t}(\cdot, s) d s\right\|_{\left(H^{1}(\Omega)\right)^{*}}^{2} d t \\
& \leq \int_{t_{k}}^{t_{k}+1}\left(\int_{t_{k}}^{t}\left\|w_{t}(\cdot, s)\right\|_{\left(H^{1}(\Omega)\right)^{*}}^{2} d s\right) \cdot\left(t-t_{k}\right) d t \\
& \leq \int_{t_{k}}^{\infty}\left\|w_{t}(\cdot, s)\right\|_{\left(H^{1}(\Omega)\right)^{*}}^{2} d s \rightarrow 0, \text { as } k \rightarrow \infty .
\end{aligned}
$$

This along with (3.11) and the continuous embedding $L^{\infty} \hookrightarrow\left(H^{1}(\Omega)\right)^{*}$ implies that

$$
\int_{t_{k}}^{t_{k}+1}\left\|w(\cdot, t)-w_{\infty}\right\|_{\left(H^{1}(\Omega)\right)^{*}}^{2} d t \rightarrow 0, \quad \text { as } k \rightarrow \infty .
$$

On the other hand, since $L^{2}(\Omega) \hookrightarrow\left(H^{1}(\Omega)\right)^{*}$ and $\int_{\Omega} w(x, t) d x=N$, we infer from Lemma 3.3 and the Poincaré inequality that

$$
\int_{0}^{\infty}\left\|w(\cdot, t)-\frac{N}{|\Omega|}\right\|_{\left(H^{1}(\Omega)\right)^{*}}^{2} d t \leq C \int_{0}^{\infty} \int_{\Omega}|\nabla w|^{2} d x d t<\infty
$$


from which it follows

$$
\int_{t_{k}}^{t_{k}+1}\left\|w(\cdot, t)-\frac{N}{|\Omega|}\right\|_{\left(H^{1}(\Omega)\right)^{*}}^{2} d t \rightarrow 0, \quad \text { as } k \rightarrow \infty .
$$

Combining (3.12) and (3.13), we derive from the uniqueness of weak limit that $w_{\infty} \equiv \frac{N}{|\Omega|}$. While, this is contradictory to (3.10) and (3.11).

Recall that we are devoted to the study of the threshold dynamics of (1.1): when $\mathcal{R}_{0}<1$, we know from Theorem 3.5 that the $\operatorname{DFE}(\tilde{S}, 0)$ is globally stable and it is unstable when $\mathcal{R}_{0}>1$ by Proposition 3.1. In the latter case, with the uniform boundedness (2.3) at hand, we are going to show that all the nontrivial solutions of (1.1) will be attracted by its $\mathrm{EE}$ in the case that the rate of disease transmission is proportional to the rate of the disease recovery, that is, $\beta(x)=r \gamma(x)$ for some positive constant $r \in(1, \infty)$ and for all $x \in \bar{\Omega}$. In this case, it follows evidently from (3.1) that $\mathcal{R}_{0}=r$ and so

$$
r>1 \Rightarrow \mathcal{R}_{0}>1, \quad r=1 \Rightarrow \mathcal{R}_{0}=1 \text { and } r<1 \Rightarrow \mathcal{R}_{0}<1 .
$$

So far, we have shown that the unique EE exists if and only if $r>1$, and $r<1$ implies DFE is globally stable, while, the DFE is neutrally stable for $r=1$. In the sequel, we shall cope with the case of $r \geq 1$ and aim to establish the global attractiveness of EE for $r>1$ and that of DFE for $r=1$. If $r>1$, the unique EE exists and is given by

$$
(\hat{S}, \hat{I})=\left(\frac{1}{r} \frac{N}{|\Omega|}, \frac{r-1}{r} \frac{N}{|\Omega|}\right) .
$$

In the case of $r>1$, by constructing a suitable Lyapunov functional, we are able to show the global stability of $(\hat{S}, \hat{I})$ for small "chemotactic" sensitivity $\chi$. In the case of $r=1$, upon a careful inspection of the reduced system, the proof of Theorem 3.5 is adaptable, and so we also have global stability for the unique $\operatorname{DFE}\left(\frac{N}{|\Omega|}, 0\right)$.

Theorem 3.6. Assume that $\beta(x)=r \gamma(x)$ for some $r \in[1, \infty)$ and for all $x \in \bar{\Omega}$.

(i) If $r>1$, then there exists a positive constant $M_{0}$ depending only on $n, \Omega, \beta, \gamma, d_{I}$ and $N$ such that whenever $0 \leq \chi<\chi_{0}:=M_{0} \sqrt{d_{S}}$, the unique classical globalin-time solution $(S, I)$ of (1.1) converges uniformly to the unique EE $(\hat{S}, \hat{I})$ in the following way:

$$
\lim _{t \rightarrow \infty}\left(\|S(\cdot, t)-\hat{S}\|_{L^{\infty}(\Omega)}+\|I(\cdot, t)-\hat{I}\|_{L^{\infty}(\Omega)}\right)=0 .
$$

That is, the unique EE ( $\hat{S}, \hat{I})$ of (1.1) is globally stable.

(ii) If $r=1$, then the unique classical global-in-time solution $(S, I)$ of (1.1) satisfies $(S, I) \rightarrow\left(\frac{N}{|\Omega|}, 0\right)$ in $C(\bar{\Omega})$ as $t \rightarrow \infty$. That is, the unique DFE $\left(\frac{N}{|\Omega|}, 0\right)$ of $(\underline{1.1})$ is globally stable.

Proof. (i) We shall use the following Lyapunov functional:

$$
V(t):=V(S, I)(t)=\int_{\Omega}\left[\left(S-\hat{S}-\hat{S} \ln \frac{S}{\hat{S}}\right)+\left(I-\hat{I}-\hat{I} \ln \frac{I}{\hat{I}}\right)\right] d x .
$$

Note that, for any $z_{0}>0$, the function $f(z)=z-z_{0}-z_{0} \ln \left(\frac{z}{z_{0}}\right), z>0$ is strictly decreasing on $\left(0, z_{0}\right)$ and is strictly increasing on $\left(z_{0}, \infty\right)$. Hence, it assumes its 
global minimum zero at $z=z_{0}$ and so $V(t) \geq 0$ for all $t \geq 0$ and $V(S, I)=0$ if and only if $(S, I)=(\hat{S}, \hat{I})$.

By (1.1), we use integration by parts to compute the time evolution of $V$ :

$$
\begin{aligned}
\frac{d V(t)}{d t}= & \int_{\Omega} \frac{S-\hat{S}}{S} S_{t} d x+\int_{\Omega} \frac{I-\hat{I}}{I} I_{t} d x \\
= & -d_{S} \hat{S} \int_{\Omega} \frac{|\nabla S|^{2}}{S^{2}} d x-\chi \hat{S} \int_{\Omega} \frac{\nabla S \cdot \nabla I}{S} d x-d_{I} \hat{I} \int_{\Omega} \frac{|\nabla I|^{2}}{I^{2}} d x \\
& -\int_{\Omega} \beta(x) I\left(\frac{S}{S+I}-\frac{1}{r}\right)\left(\frac{S-\hat{S}}{S}-\frac{I-\hat{I}}{I}\right) d x \\
= & -\hat{S} \int_{\Omega}\left(\sqrt{d_{S}} \frac{\nabla S}{S}-\frac{\chi}{2 \sqrt{d_{S}}} \nabla I\right)^{2} d x-\hat{S} \int_{\Omega}\left((r-1) d_{I}-\frac{I^{2} \chi^{2}}{4 d_{S}}\right) \frac{|\nabla I|^{2}}{I^{2}} d x \\
& -\int_{\Omega} \frac{\beta(x) S I^{2}}{(S+I)(\hat{S}+\hat{I})}\left(\frac{\hat{I}}{I}-\frac{\hat{S}}{S}\right)^{2} d x \\
= & -\hat{S} \int_{\Omega}\left(d_{S}-\frac{I^{2} \chi^{2}}{4 d_{I}(r-1)}\right) \frac{|\nabla S|^{2}}{S^{2}} d x-\int_{\Omega}\left(\sqrt{d_{I} \hat{I}} \frac{\nabla I}{I}-\frac{\hat{S} I \chi}{2 \sqrt{d_{I} \hat{I}} \frac{\nabla S}{S}}\right)^{2} d x \\
& -\frac{1}{r} \int_{\Omega} \frac{\beta(x) \hat{S}}{(S+I) S}[(r-1) S-I]^{2} d x,
\end{aligned}
$$

where we have used the assumption $\beta(x)=r \gamma(x)$ to entail

$$
\frac{1}{r}=\frac{\hat{S}}{\hat{S}+\hat{I}}, \quad \hat{I}=(r-1) \hat{S} .
$$

Now, noticing that $\|I(\cdot, t)\|_{L^{\infty}}\left(\leq M_{I}\left(n, \Omega, d_{I}, \gamma_{*}, \beta^{*}, S_{0}, I_{0}\right)\right)$ is uniformly bounded with respect to $\chi$ by (2.5), we see, if

$$
0 \leq \chi<\chi_{0}:=\frac{2}{M_{I}\left(n, \Omega, d_{I}, \gamma_{*}, \beta^{*}, S_{0}, I_{0}\right)} \sqrt{(r-1) d_{S} d_{I}},
$$

then, with the boundedness of $S$ and $I$ as in (2.3), we infer from (3.16) and (3.15) there exists $c_{0}>0$ such that

$$
\frac{d V(t)}{d t} \leq-c_{0}\left\{\int_{\Omega}|\nabla S|^{2} d x+\int_{\Omega}|\nabla I|^{2} d x+\int_{\Omega}[(r-1) S-I]^{2} d x\right\} .
$$

Because of $V(t) \geq 0$, an integration of (3.17) from 1 to $t$ shows

$$
\int_{1}^{t}\left[\int_{\Omega}|\nabla S|^{2} d x+\int_{\Omega}|\nabla I|^{2} d x+\int_{\Omega}[(r-1) S-I]^{2} d x\right] d s \leq \frac{V(1)}{c_{0}}<\infty, \forall t>t_{0},
$$

which yields trivially

$$
\int_{1}^{\infty}\left[\int_{\Omega}|\nabla S|^{2} d x+\int_{\Omega}|\nabla I|^{2} d x+\int_{\Omega}[(r-1) S-I]^{2} d x\right] d s \leq \frac{V(1)}{c_{0}}<\infty .
$$

To proceed further, we need the integrand inside the big square bracket in (3.18) to be uniformly bounded and uniformly continuous. For this purpose, we need further Hölder type regularity for $S$ and $I$. To achieve this, we rewrite the $S$-equation as

$$
S_{t}=\nabla \cdot(A(x, t, \nabla S))+B(x, t)
$$


with

$A(x, t, \xi)=d_{S} \xi+\chi S(x, t) \nabla I(x, t), \quad B(x, t)=-\beta(x) \frac{S(x, t) I(x, t)}{S(x, t)+I(x, t)}+\gamma(x) I(x, t)$.

Then, thanks to the boundedness information provided in Theorem 2.3, it is an easy matter to check, for some $c_{i}>0, i=1,2,3$, that

$$
\left\{\begin{array}{l}
A(x, t, \xi) \cdot \xi \geq \frac{d_{S}}{2}|\xi|^{2}-c_{1}, \quad|A(x, t, \xi)| \leq d_{S}|\xi|+c_{2}, \quad \forall(x, t, \xi) \in \Omega \times(0, \infty) \times \mathbb{R}^{n}, \\
|B(x, t)| \leq c_{3}, \quad \forall(x, t, \xi) \in \Omega \times(0, \infty) \times \mathbb{R}^{n} .
\end{array}\right.
$$

Now, $S$ and $I$ are bounded in $\Omega \times(0, \infty)$, applying the Hölder estimates for parabolic equations (cf. [32, Theorem 1.3]) and then employing the standard parabolic Schauder theory (cf. [14, 23]), we see there exist $\theta \in(0,1)$ and $C>0$ such that

$$
\|S\|_{C^{2+\theta, 1+\frac{\theta}{2}(\bar{\Omega} \times[t, t+1])}}+\|I\|_{C^{2+\theta, 1+\frac{\theta}{2}(\bar{\Omega} \times[t, t+1])}} \leq C, \quad \forall t \geq 1 .
$$

Thus, the integrand inside the big square bracket in (3.18) is uniformly bounded and uniformly continuous, and so (3.18) entails

$$
\lim _{t \rightarrow \infty}\left(\int_{\Omega}|\nabla S|^{2} d x+\int_{\Omega}|\nabla I|^{2} d x\right)=0
$$

and

$$
\lim _{t \rightarrow \infty} \int_{\Omega}[(r-1) S-I]^{2} d x=0 .
$$

The latter along with the conservation of $S+I$ as in (1.4) gives

$$
\lim _{t \rightarrow \infty} \bar{S}:=\lim _{t \rightarrow \infty} \frac{1}{|\Omega|} \int_{\Omega} S d x=\hat{S}, \quad \lim _{t \rightarrow \infty} \bar{I}:=\lim _{t \rightarrow \infty} \frac{1}{|\Omega|} \int_{\Omega} I d x=\hat{I} .
$$

Recalling from the Poincaré inequality, we have

$$
\begin{aligned}
& \int_{\Omega}\left[(S-\hat{S})^{2}+(I-\hat{I})^{2}\right] d x \\
& =\int_{\Omega}\left[(S-\bar{S})^{2}+(I-\bar{I})^{2}\right] d x+|\Omega|\left[(\bar{S}-\hat{S})^{2}+(\bar{I}-\hat{I})^{2}\right] \\
& \leq \frac{1}{\lambda_{1}} \int_{\Omega}\left(|\nabla S|^{2}+|\nabla I|^{2}\right) d x+|\Omega|\left[(\bar{S}-\hat{S})^{2}+(\bar{I}-\hat{I})^{2}\right] .
\end{aligned}
$$

This in conjunction with (3.20) and (3.21) readily shows

$$
\lim _{t \rightarrow \infty} \int_{\Omega}\left[(S-\hat{S})^{2}+(I-\hat{I})^{2}\right] d x=0 .
$$

Finally, in view of the Gagliardo-Nirenberg inequality, we derive from (3.19) that

$$
\begin{aligned}
& \|S-\hat{S}\|_{L^{\infty}(\Omega)}+\|I-\hat{I}\|_{L^{\infty}(\Omega)} \\
\leq & C_{G N}\left(\|S-\hat{S}\|_{W^{1, \infty}(\Omega)}^{\frac{n}{n+2}}\|S-\hat{S}\|_{L^{2}(\Omega)}^{\frac{2}{n+2}}+\|I-\hat{I}\|_{W^{1, \infty}(\Omega)}^{\frac{n}{n+2}}\|I-\hat{I}\|_{L^{2}(\Omega)}^{\frac{2}{n+2}}\right) \\
\leq & C\left(\|S-\hat{S}\|_{L^{2}(\Omega)}^{\frac{2}{n+2}}+\|I-\hat{I}\|_{L^{2}(\Omega)}^{\frac{2}{n+2}}\right)
\end{aligned}
$$

which coupled with (3.22) evidently gives rise to (3.14). 
(ii) Since $\beta(x)=\gamma(x)$, system (1.1) reduces to

$$
\begin{cases}S_{t}=d_{S} \Delta S+\chi \nabla \cdot(S \nabla I)+\beta(x) \frac{I^{2}}{S+I}, & x \in \Omega, t>0, \\ I_{t}=d_{I} \Delta I-\beta(x) \frac{I^{2}}{S+I}, & x \in \Omega, t>0, \\ \frac{\partial S}{\partial \nu}=\frac{\partial I}{\partial \nu}=0, & x \in \partial \Omega, t>0, \\ (S(x, 0), I(x, 0))=\left(S_{0}(x), I_{0}(x)\right), & x \in \Omega .\end{cases}
$$

Then the boundedness of $S+I$ in (2.3) shows that

$$
I_{t}=d_{I} \Delta I-\beta(x) \frac{I^{2}}{S+I} \leq d_{I} \Delta I-\beta_{*} \frac{I^{2}}{M}=d_{I} \Delta I-\delta I^{2},
$$

where $\beta_{*}=\min _{\bar{\Omega}} \beta$ and $\delta=\beta_{*} / M>0$. Now, we consider the following ODE

$$
\left\{\begin{array}{l}
\bar{I}^{\prime}=-\delta \bar{I}^{2}, \quad t>0 \\
\bar{I}(0)=\left\|I_{0}\right\|_{L^{\infty}(\Omega)}>0
\end{array}\right.
$$

Upon an application of the maximum principle and direct calculations, we have

$$
I(\cdot, t) \leq \bar{I}(t)=\frac{1}{1 / \bar{I}(0)+\delta t} \rightarrow 0, \quad \text { as } t \rightarrow \infty .
$$

To prove the desired convergence of $S$, one can proceed similarly to the proof of Theorem 3.5. In fact, as in (3.7), we first observe that the algebraic decay (3.24) is sufficient for us to infer

$$
\int_{0}^{\infty} \int_{\Omega}|\nabla I|^{2} d x d t<\infty
$$

On the other hand, using (3.23), we calculate

$$
\begin{aligned}
\frac{1}{2} \frac{d}{d t} \int_{\Omega} S^{2} d x & =-d_{S} \int_{\Omega}|\nabla S|^{2} d x-\chi \int_{\Omega} S \nabla I \cdot \nabla S d x+\int_{\Omega} \beta \frac{S I^{2}}{S+I} d x \\
& \leq-d_{S} \int_{\Omega}|\nabla S|^{2} d x+\frac{d_{S}}{2} \int_{\Omega}|\nabla S|^{2} d x+\frac{\chi^{2}}{2 d_{S}} \int_{\Omega} S^{2}|\nabla I|^{2} d x+\beta^{*} \int_{\Omega} I^{2} d x \\
& \leq-\frac{d_{S}}{2} \int_{\Omega}|\nabla S|^{2} d x+\frac{\chi^{2}}{2 d_{S}}\|S(\cdot, t)\|_{L^{\infty}(\Omega)}^{2} \int_{\Omega}|\nabla I|^{2} d x+\beta^{*} \int_{\Omega} I^{2} d x .
\end{aligned}
$$

Then (3.24) and (3.25) enable us to conclude that

$$
\int_{0}^{\infty} \int_{\Omega}|\nabla S|^{2} d x d t<\infty
$$

With these key ingredients obtained, the remaining proof follows along the lines of the argument of Theorem 3.5, and hence we omit the details.

For small $\chi>0$, Theorem 3.6 conveys to us that system (1.1) is uniformly persistent when $\mathcal{R}_{0}>1$. Equipped with the "ultimately uniformly boundedness" (2.4), we can indeed adapt the arguments of [31, Theorem 3.3], developed by Magal and Zhao (see [27, Theorem 4.5] or [42, Chapter 13]), to deduce that system (1.1) is indeed uniformly persistent for any $\chi>0$. Specifically, we have

Theorem 3.7. Let $\left(u_{0}, v_{0}\right)$ obey (1.3) and the basic reproduction number $\mathcal{R}_{0}>1$. Then system (1.1) is uniformly persistent, i.e., there exists some $\eta>0$, independent of $\left(u_{0}, v_{0}\right)$, such that

$$
\liminf _{t \rightarrow \infty} S(x, t) \geq \eta \text { and } \liminf _{t \rightarrow \infty} I(x, t) \geq \eta \text { uniformly for } x \in \bar{\Omega} .
$$


Furthermore, there exists at least an $E E(S, I)$ of (1.1) fulfilling

$$
\int_{\Omega}[S(x)+I(x)] d x=N .
$$

\section{Existence And Uniqueness of EE}

Although Theorem 3.7 provides us with the existence of EE when $\mathcal{R}_{0}>1$, the uniqueness is unclear. In this section, we shall discuss the existence of EE via a different method. In view of the special reaction terms in system (1.1), we can reduce the elliptic problem of (1.1) to a single equation, for which the existence of a positive solution can be obtained by a pure PDE approach. Moreover, this technique allows one to deal with the uniqueness and the computations here are also crucial for the forthcoming section where we discuss the asymptotic behavior of EE for small $d_{S}>0$. Hence, in the following, we focus on the steady state system associated with (1.1):

$$
\begin{cases}d_{S} \Delta S+\chi \nabla \cdot(S \nabla I)-\beta(x) \frac{S I}{S+I}+\gamma(x) I=0, & x \in \Omega, \\ d_{I} \Delta I+\beta(x) \frac{S I}{S+I}-\gamma(x) I=0, & x \in \Omega, \\ \frac{\partial S}{\partial \nu}=\frac{\partial I}{\partial \nu}=0, & x \in \partial \Omega, \\ \int_{\Omega}[S(x)+I(x)] d x=N . & \end{cases}
$$

Recall that an $\mathrm{EE}(S, I)$ is a nonnegative solution of (4.1) with $I \not \equiv 0$ on $\Omega$. A direct application of the strong maximum principle and Hopf boundary point lemma asserts $S, I>0$ on $\bar{\Omega}$.

Adding the first two PDEs in (4.1), we see that

$$
\nabla \cdot\left(d_{S} \nabla S+d_{I} \nabla I+\chi S \nabla I\right)=0
$$

or equivalently

$$
\nabla \cdot\left[\left(1+\frac{\chi}{d_{I}} S\right) \nabla\left(\frac{d_{S}}{\chi} \ln \left(1+\frac{\chi}{d_{I}} S\right)+I\right)\right]=0 .
$$

We claim that

$$
\frac{d_{S}}{\chi} \ln \left(1+\frac{\chi}{d_{I}} S\right)+I \equiv \kappa
$$

for some positive constant $\kappa$. In fact, upon setting

$$
w=\frac{d_{S}}{\chi} \ln \left(1+\frac{\chi}{d_{I}} S\right)+I,
$$

we get from (4.2) that

$$
\nabla \cdot\left[\left(1+\frac{\chi}{d_{S}} S\right) \nabla w\right]=0
$$

As a result,

$$
\begin{aligned}
\nabla \cdot\left[w\left(1+\frac{\chi}{d_{S}} S\right) \nabla w\right] & =w \nabla \cdot\left[\left(1+\frac{\chi}{d_{S}} S\right) \nabla w\right]+\left(1+\frac{\chi}{d_{S}} S\right)|\nabla w|^{2} \\
& =\left(1+\frac{\chi}{d_{S}} S\right)|\nabla w|^{2} .
\end{aligned}
$$

Upon an integration, one sees that $w$ must be constant and hence (4.3) holds. 
Let

$$
\tilde{I}=\frac{I}{\kappa} \text { and } \tilde{S}=\frac{1}{\kappa \chi} \ln \left(1+\frac{\chi}{d_{I}} S\right) .
$$

Then (4.3) gives rise to

$$
d_{S} \tilde{S}+\tilde{I}=1
$$

or

from which it follows

$$
\frac{d_{S}}{\kappa \chi} \ln \left(1+\frac{\chi}{d_{I}} S\right)+\tilde{I}=1
$$

$$
S=\frac{d_{I}}{\chi}\left[\exp \left\{\frac{\kappa \chi}{d_{S}}(1-\tilde{I})\right\}-1\right]=: g(\tilde{I}) .
$$

Define

$$
f(x, \tilde{I})=\beta(x) \frac{g(\tilde{I})}{g(\tilde{I})+\kappa \tilde{I}}-\gamma(x) .
$$

According to the $I$-equation, (4.4) and (4.7), it can be easily seen that $\tilde{I}$ solves

$$
\begin{cases}d_{I} \Delta \tilde{I}+\tilde{I} f(x, \tilde{I})=0, & x \in \Omega, \\ \frac{\partial \tilde{I}}{\partial \nu}=0, & x \in \partial \Omega .\end{cases}
$$

In addition, the integral constraint (4.1) and (4.4) show

$$
N=\int_{\Omega}(S+I) d x=\frac{d_{I}}{\chi} \int_{\Omega}\left(e^{\kappa \chi \tilde{S}}-1\right) d x+\kappa \int_{\Omega} \tilde{I} d x
$$

These discussions yield equivalent descriptions of the equilibrium problem (4.1).

Lemma 4.1. A pair $(S, I)$ is a positive solution of (4.1) if and only if $(\tilde{S}, \tilde{I})$ is a positive solution of (4.9) and (4.5) with $\kappa$ being the unique positive constant determined by (4.10).

Thanks to the conservation of total population, we have reduced the system (4.1) to a single equation (4.9), and then we can easily establish the existence and uniqueness of EE for the cross-diffusive SIS model (1.1).

Theorem 4.2. When $\mathcal{R}_{0}>1$, the cross-diffusive SIS model (1.1) has a unique EE.

Proof. It is enough to show that (4.9) admits a unique positive solution $\tilde{I}$ with $\tilde{I}<1$. If so, one can solve $\tilde{S}(>0)$ from (4.5), and then $\kappa>0$ is uniquely determined via (4.10). The assumption $\mathcal{R}_{0}>1$ entails $\lambda^{*}<0$, where $\lambda^{*}$ is the principal eigenvalue of the eigenvalue problem (3.2). Let $\phi^{*}>0$ be the corresponding principal eigenfunction. Direct calculations imply that $\underline{I}=\epsilon \phi^{*}$ and $\bar{I} \equiv 1$ is a pair of suband super-solutions of (4.9), provided $\epsilon>0$ is chosen to be sufficiently small. Thus, there exists some $\tilde{I} \in[\underline{I}, \bar{I}]$. As a result, $0<\tilde{I} \leq 1$ on $\bar{\Omega}$.

We now claim $0<\tilde{I}<1$ on $\bar{\Omega}$. In fact, let $\tilde{I}\left(x_{0}\right)=\max _{\bar{\Omega}} \tilde{I}$. Then the maximum principle [26, Proposition 2.2] applied to (4.9) entails that

$$
\beta\left(x_{0}\right) \frac{g\left(\tilde{I}\left(x_{0}\right)\right)}{g\left(\tilde{I}\left(x_{0}\right)\right)+\kappa \tilde{I}\left(x_{0}\right)} \geq \gamma\left(x_{0}\right)>0 .
$$

By the definition of $g$ in (4.7) and the fact that $0<\tilde{I} \leq 1$, we have $g(\tilde{I}) \geq 0$. Then the above inequality yields $g\left(\tilde{I}\left(x_{0}\right)\right)>0$, which in turn shows that $\tilde{I}(x) \leq \tilde{I}\left(x_{0}\right)<1$ for $x \in \bar{\Omega}$. 
Finally, thanks to the fact $\tilde{I} \in(0,1)$, we simply calculate from (4.7) and (4.8) that $\frac{\partial f}{\partial \tilde{I}}(x, \tilde{I})<0$ for $x \in \bar{\Omega}$. This enables us to deduce the uniqueness of $\tilde{I}$; see the detailed argument in the proof of [프, Lemma 3.3].

\section{Asymptotic Behavior of EE AS $d_{S} \rightarrow 0$}

In this section, we shall study the effect of motility of susceptible population. That is, we will investigate the asymptotic behavior of $\mathrm{EE}$ as $d_{S} \rightarrow 0$. We always assume $\mathcal{R}_{0}>1$ so that (1.1) possesses a unique EE by Theorem 4.2 . Depending on whether or not $\beta(x)-\gamma(x)$ changes sign, we consider two different cases.

We first present a simple lemma, providing the asymptotic behavior of $\tilde{I}$ defined via (4.4) for small $d_{S}>0$.

Lemma 5.1. If $\mathcal{R}_{0}>1$, up to a subsequence of $d_{S} \rightarrow 0$, it holds $\tilde{I} \rightarrow \tilde{I}^{*}$ in $C^{1}(\bar{\Omega})$ for some $\tilde{I}^{*} \in C^{1}(\bar{\Omega})$ with $0<\tilde{I}^{*}(x) \leq 1$ for $x \in \bar{\Omega}$ and $\partial \tilde{I}^{*} / \partial \nu=0$ on $\partial \Omega$.

Proof. First, it follows from the definition of $f$ in (4.8) that

$$
\|f(x, \tilde{I})\|_{L^{\infty}(\Omega)} \leq \beta^{*}+\gamma^{*}, \quad \forall d_{S}>0
$$

An application of the Harnack inequality [28, Lemma 2.2] to (4.9) gives

$$
\max _{\bar{\Omega}} \tilde{I} \leq C \min _{\bar{\Omega}} \tilde{I}
$$

for some positive constant $C$ independent of $d_{S}>0$.

Next, we have shown that $0<\tilde{I}<1$ and so $\tilde{I}$ is uniformly bounded for $d_{S}>0$. Hence, applying the standard $L^{p}$-estimates to (4.9) and the Sobolev embedding theorem, we infer that the $C^{1+\alpha}(\bar{\Omega})$-bound of $\tilde{I}$ is also independent of $d_{S}>0$ for some $\alpha \in(0,1)$. Thus, after passing to a subsequence of $d_{S} \rightarrow 0$, it holds

$$
\tilde{I} \rightarrow \tilde{I}^{*} \geq 0 \text { in } C^{1}(\bar{\Omega}), \quad \text { as } d_{S} \rightarrow 0
$$

for some $\tilde{I}^{*} \in C^{1}(\bar{\Omega})$. This $C^{1}$-convergence enforces that $\tilde{I}$ fulfills the homogeneous Neumann boundary condition on $\partial \Omega$. Furthermore, by (5.1), it follows

$$
\tilde{I}^{*}>0 \text { on } \bar{\Omega} \quad \text { or } \quad \tilde{I}^{*} \equiv 0 .
$$

On the other hand, it is easy to check from the definitions of $g$ in (4.7) and $f$ in (4.8) that both $g$ and $f$ are decreasing with respect to $d_{S}>0$. Then the same argument as [3. Lemma 4.1] shows that $\tilde{I}$ is in fact a decreasing function of $d_{S}$. Consequently, it must hold $\tilde{I}^{*}>0$ over $\bar{\Omega}$. This finishes the proof.

Now, we are ready to present the asymptotic behavior of EE when $\beta>\gamma$ on $\bar{\Omega}$.

Theorem 5.2. Suppose $\beta(x)>\gamma(x)$ on $\bar{\Omega}$. Then as $d_{S} \rightarrow 0$, any $E E(S, I)$ of the model (1.1) satisfies $(S, I) \rightarrow\left(S^{*}, I^{*}\right)$ uniformly on $\bar{\Omega}$ with

$$
S^{*}=\frac{\gamma(x)}{\beta(x)-\gamma(x)} I^{*} \quad \text { and } \quad I^{*}=\frac{N}{\int_{\Omega} \frac{\beta(x)}{\beta(x)-\gamma(x)} d x} .
$$

Proof. We first notice that $\beta>\gamma$ on $\bar{\Omega}$ is sufficient to guarantee $\mathcal{R}_{0}>1$ and hence a unique EE $(S, I)$ of (1.1) exists by Theorem 4.2. The $I$-equation reads as

$$
\begin{cases}d_{I} \Delta I+\left(\beta \frac{S}{S+I}-\gamma\right) I=0, & x \in \Omega, \\ \frac{\partial I}{\partial \nu}=0, & x \in \partial \Omega\end{cases}
$$


Observing

$$
\left\|\beta \frac{S}{S+I}-\gamma\right\|_{L^{\infty}(\Omega)} \leq \beta^{*}+\gamma^{*}, \quad \forall d_{S}>0,
$$

we obtain from the Harnack inequality that

$$
\max _{\bar{\Omega}} I \leq C \min _{\bar{\Omega}} I
$$

for some positive constant $C$ independent of $d_{S}>0$. Since $\int_{\Omega} I d x \leq N$, we once again apply (5.4) to end up with

$$
\max _{\bar{\Omega}} I \leq C \min _{\bar{\Omega}} I \leq \frac{C}{|\Omega|} \int_{\Omega} I d x \leq \frac{C N}{|\Omega|} .
$$

Thus, the $L^{\infty}$-bound of $I$ is independent of $d_{S}>0$. The same argument leading to (5.2) shows, after passing to a subsequence of $d_{S} \rightarrow 0$, that

$$
I \rightarrow I^{*} \geq 0 \text { in } C^{1}(\bar{\Omega}), \quad \text { as } d_{S} \rightarrow 0
$$

for some $I^{*} \in C^{1}(\bar{\Omega})$. Moreover, the Harnack inequality (5.4) implies

$$
I^{*}>0 \quad \text { on } \bar{\Omega} \quad \text { or } \quad I^{*} \equiv 0 \text { on } \bar{\Omega} \text {. }
$$

We now expand out the cross-diffusive term in the $S$-equation and use the $I$-equation to discover that $S$ fulfills

$$
\begin{cases}d_{S} \Delta S+\chi \nabla S \cdot \nabla I=I\left(1+\frac{\chi S}{d_{I}}\right)\left(\beta \frac{S}{S+I}-\gamma\right), & x \in \Omega \\ \frac{\partial S}{\partial I}=0, & x \in \partial \Omega .\end{cases}
$$

Let $S\left(x_{0}\right)=\max _{\bar{\Omega}} S$. Then the maximum principle in [26, Proposition 2.2] entails

$$
I\left(x_{0}\right)\left(1+\frac{\chi S\left(x_{0}\right)}{d_{I}}\right) \frac{\left(\beta\left(x_{0}\right)-\gamma\left(x_{0}\right)\right) S\left(x_{0}\right)-\gamma\left(x_{0}\right) I\left(x_{0}\right)}{S\left(x_{0}\right)+I\left(x_{0}\right)} \leq 0,
$$

from which it follows

$$
\max _{\bar{\Omega}} S=S\left(x_{0}\right) \leq \frac{\gamma\left(x_{0}\right)}{\beta\left(x_{0}\right)-\gamma\left(x_{0}\right)} I\left(x_{0}\right) \leq\left(\max _{\bar{\Omega}} \frac{\gamma}{\beta-\gamma}\right)\|I\|_{L^{\infty}(\Omega)} .
$$

This in conjunction with (5.6) and (5.5) forces $I^{*}>0$, since otherwise both $S$ and $I$ are small for sufficiently small $d_{S}>0$, contradicting the prescribed mass conservation $\int_{\Omega}[S(x)+I(x)] d x=N$.

We now claim that $\tilde{I}^{*} \equiv 1$, where $\tilde{I}^{*}$ is given in Lemma 5.1. Suppose not, then according to Lemma 5.1 , we have

$$
\int_{\Omega}\left(1-\tilde{I}^{*}\right) d x>0
$$

In view of the relation of $S$ and $\tilde{I}$ in (4.6), for small $d_{S}>0$, we deduce

$$
\begin{aligned}
0<\frac{1}{2} \int_{\Omega}\left(1-\tilde{I}^{*}\right) d x<\int_{\Omega}(1-\tilde{I}) d x & =\frac{d_{S}}{\kappa \chi} \int_{\Omega} \ln \left(1+\frac{\chi}{d_{I}} S\right) d x \\
& \leq \frac{d_{S}}{\kappa \chi} \int_{\Omega} \frac{\chi}{d_{I}} S d x \leq \frac{d_{S}}{\kappa d_{I}} N
\end{aligned}
$$

from which it follows that $\kappa \rightarrow 0$ as $d_{S} \rightarrow 0$. As a result, $I \rightarrow 0$ as $d_{S} \rightarrow 0$ due to (4.3). However, this is a contradiction to $I^{*}>0$, as we have just proved. Thus, we 
must have $\tilde{I}^{*} \equiv 1$. Consequently, since $I \rightarrow I^{*}$ in $C^{1}(\bar{\Omega})$ as $d_{S} \rightarrow 0$ and $I=\kappa \tilde{I}$ for positive constant $\kappa$, then $I^{*}$ is necessarily a positive constant.

Since $I \rightarrow I^{*} \equiv$ const $>0$ in $C^{1}(\bar{\Omega})$ (in turn $|\nabla I| \rightarrow 0$ uniformly on $\bar{\Omega}$ ) as $d_{S} \rightarrow 0$, a standard singular perturbation argument (see, for instance, [13, Lemma 2.4] or [19]) applied to (5.7) yields that

$$
S(x) \rightarrow S^{*}(x)=\frac{\gamma(x)}{\beta(x)-\gamma(x)} I^{*} \quad \text { uniformly on } \bar{\Omega},
$$

as $d_{S} \rightarrow 0$. Then the conservation of total population

$$
\int_{\Omega}\left(S^{*}+I^{*}\right) d x=N
$$

simply gives (5.3). The uniqueness of $\left(S^{*}, I^{*}\right)$ says that all the above limits hold without passing to a subsequence of $d_{S} \rightarrow 0$.

In the sequel, besides $\mathcal{R}_{0}>1$, we shall assume that the set $\{x \in \bar{\Omega}: \beta(x)<\gamma(x)\}$ is nonempty, which in fact indicates that $\beta(x)-\gamma(x)$ must change sign. Note that all of $S, I$ and $\kappa>0$ in (4.3) depend on $d_{S}$. Hence, to determine their asymptotics as $d_{S} \rightarrow 0$, we shall start with the limiting function of $\tilde{I}$, i.e., $\tilde{I}^{*} \in(0,1]$. To further study the limiting function $\tilde{I}^{*}$, we need to determine where $0<\tilde{I}^{*}<1$ and where $\tilde{I}^{*}=1$. For these purposes, we define

$$
H^{-}=\{x \in \Omega: \beta(x)<\gamma(x)\} \quad \text { and } \quad H^{+}=\{x \in \Omega: \beta(x)>\gamma(x)\}
$$

and

$$
J^{-}=\left\{x \in \bar{\Omega}: 0<\tilde{I}^{*}(x)<1\right\} \quad \text { and } \quad J^{+}=\left\{x \in \bar{\Omega}: \tilde{I}^{*}(x)=1\right\} .
$$

Lemma 5.3. Suppose that $\mathcal{R}_{0}>1$ and $\{x \in \bar{\Omega}: \beta(x)<\gamma(x)\} \neq \emptyset$. Then the following statements hold.

(i) $H^{-} \subset J^{-}$;

(ii) after passing to a subsequence of $d_{S} \rightarrow 0, \kappa \rightarrow 0$ and $I \rightarrow 0$ in $C^{1}(\bar{\Omega})$;

(iii) after passing to a subsequence of $d_{S} \rightarrow 0, \kappa / d_{S} \rightarrow M>0$, where $M$ is the unique number satisfying

$$
\int_{\Omega} e^{\chi\left(1-\tilde{I}^{*}(x)\right) M} d x=\frac{N \chi}{d_{I}}+|\Omega|
$$

and

$$
S(x) \rightarrow S^{*}(x):=\frac{d_{I}}{\chi}\left[e^{\chi\left(1-\tilde{I}^{*}(x)\right) M}-1\right] \text { in } C^{1}(\bar{\Omega}) .
$$

Proof. (i) can be proved by using an indirect argument as in [3, Lemma 4.3].

(ii) Firstly, it follows from (4.6) that

$$
\frac{\kappa \chi}{d_{S}} \int_{\Omega}(1-\tilde{I}) d x=\int_{\Omega} \ln \left(1+\frac{\chi}{d_{I}} S\right) d x \leq \int_{\Omega} \frac{\chi}{d_{I}} S d x \leq \frac{\chi}{d_{I}} N .
$$

On the other hand, as $d_{S} \rightarrow 0$, the item (i) implies

$$
\int_{\Omega}(1-\tilde{I}) d x \rightarrow \int_{\Omega}\left(1-\tilde{I}^{*}\right) d x \geq \int_{H^{-}}\left(1-\tilde{I}^{*}\right) d x>0 .
$$

As a result, for small $d_{S}>0$, it holds

$$
\int_{\Omega}(1-\tilde{I}) d x \geq \frac{1}{2} \int_{H^{-}}\left(1-\tilde{I}^{*}\right) d x>0
$$


This, together with (5.12), indicates that for small $d_{S}>0$,

$$
0<\frac{\kappa}{2 d_{S}} \int_{H^{-}}\left(1-\tilde{I}^{*}\right) d x \leq \frac{N}{d_{I}}
$$

This forces $\kappa \rightarrow 0$ as $d_{S} \rightarrow 0$, and so (4.3) ensures $I \leq \kappa \rightarrow 0$ uniformly on $\bar{\Omega}$ as $d_{S} \rightarrow 0$. Since $\tilde{I} \rightarrow \tilde{I}^{*}$ in $C^{1}(\bar{\Omega})$ as $d_{S} \rightarrow 0$ by Lemma 5.1, it follows from $I=\kappa \tilde{I}$ that $I \rightarrow 0$ in $C^{1}(\bar{\Omega})$ as $d_{S} \rightarrow 0$.

(iii) Using (4.7), the fact that $\tilde{I} \rightarrow \tilde{I}^{*}$ and (i), we get

$$
\begin{aligned}
N=\int_{\Omega} I d x+\int_{\Omega} S d x & =\int_{\Omega} I d x+\frac{d_{I}}{\chi} \int_{\Omega}\left[e^{\frac{\kappa \chi}{d S}(1-\tilde{I})}-1\right] d x \\
& \geq \frac{d_{I}}{2 \chi} \int_{H^{-}}\left[e^{\frac{\kappa \chi}{d_{S}}\left(1-\tilde{I}^{*}\right)}-1\right] d x>0
\end{aligned}
$$

for small $d_{S}>0$. This first tells us that $\kappa / d_{S}$ is bounded for small $d_{S}>0$. Thus, after further passing to a subsequence of $d_{S} \rightarrow 0$ if necessary, we can assume that $\kappa / d_{S} \rightarrow M \geq 0$. Moreover, by sending $d_{S} \rightarrow 0$ in (5.13) and using (ii), we see that $M$ is determined via (5.10). Obviously, such $M$ is unique and $M>0$. Finally, (5.11) can be seen from (4.7) and Lemma 5.1.

Remark 5.4. Our results here match those of [3, Lemma 4.5] with $\chi=0$. In fact, if formally letting $\chi \rightarrow 0$ in (4.6), we obtain that

$$
\frac{d_{S} S}{\kappa d_{I}}+\tilde{I}=1
$$

Upon an integration and using the conservative property of total population, we have

$$
\frac{d_{S}}{\kappa d_{I}}\left(N-\int_{\Omega} I d x\right)=\int_{\Omega}(1-\tilde{I}) d x
$$

Since $I \rightarrow 0$ and $\tilde{I} \rightarrow \tilde{I}^{*}$ as $d_{S} \rightarrow 0$, it follows that

$$
\frac{\kappa}{d_{S}} \rightarrow N^{*}:=\frac{N}{d_{I} \int_{\Omega}\left(1-\tilde{I}^{*}\right) d x} .
$$

Furthermore, if we let $\chi \rightarrow 0$ in (5.11), we formally obtain that

$$
S \rightarrow S^{*}=d_{I}\left(1-\tilde{I}^{*}\right) N^{*}
$$

Those are the asympotics proved in [3, Lemma 4.5].

In view of (5.9) and (5.11), it holds

$$
J^{-}=\left\{x \in \bar{\Omega}: S^{*}(x)>0\right\} \quad \text { and } \quad J^{+}=\left\{x \in \bar{\Omega}: S^{*}(x)=0\right\} .
$$

Using the same arguments as those in [3, Lemmas 4.6 and 4.7], one can further prove the following properties of the sets $J^{+}$and $J^{-}$.

Lemma 5.5. Suppose that $\mathcal{R}_{0}>1$ and $\{x \in \bar{\Omega}: \beta(x)<\gamma(x)\} \neq \emptyset$. Then $\emptyset \neq$ $J^{+} \subset \overline{H^{+}}$and the set $J^{+}$has positive measure. If we further assume that the set $H^{0}=\{x \in \Omega: \beta(x)=\gamma(x)\}$ consists of finitely many disjoint $C^{1}$-surfaces (or finitely many points if $n=1$, each of which is a simple root of $\beta-\gamma)$. Then $\overline{H^{-}}$is a proper subset of $J^{-}$. Moreover, $\tilde{I}^{*} \in C^{2}\left(J^{-}\right)$satisfies $d_{I} \Delta \tilde{I}^{*}+(\beta-\gamma) \tilde{I}^{*}=0$ on $J^{-}$.

We summarize the findings above in the following theorem.

Theorem 5.6. Suppose that $\mathcal{R}_{0}>1$ and $\{x \in \bar{\Omega}: \beta(x)<\gamma(x)\} \neq \emptyset$. 
(i) As $d_{S} \rightarrow 0$, any $E E(S, I)$ of the cross-diffusive SIS model (1.1) satisfies $(S, I) \rightarrow\left(S^{*}, 0\right)$ in $C^{1}(\bar{\Omega})$ with $S^{*}$ satisfying

$$
S^{*} \geq 0, \quad \frac{\partial S^{*}}{\partial \nu}=0 \text { on } \partial \Omega \quad \text { and } \quad \int_{\Omega} S^{*}(x) d x=N .
$$

(ii) The set $J^{-}:=\left\{x \in \bar{\Omega}: S^{*}(x)>0\right\}$ contains $H^{-}$;

(iii) The set $J^{+}:=\left\{x \in \bar{\Omega}: S^{*}(x)=0\right\}$ has positive measure and it is contained in $\overline{H^{+}}$;

(iv) If we further assume that the set $H^{0}=\{x \in \Omega: \beta(x)=\gamma(x)\}$ consists of finitely many disjoint $C^{1}$-surfaces (or finitely many points if $n=1$, each of which is a simple root of $\beta-\gamma)$, then $\overline{H^{-}} \subset J^{-}$and the set $J^{-} \backslash H^{-}$has positive measure.

\section{The Model with Varying Total Population}

In this section, we briefly discuss the model (1.2) with cross-diffusion and linear source. We first notice that the global existence and boundedness of solutions have been included in Theorem 2.3. Next, we plan to discuss the global stability of the equilibria of system (1.2). It can be easily seen that the unique DFE is given by $(\tilde{S}, 0)$, where $\tilde{S}$ is the unique positive solution of

$$
\begin{cases}d_{S} \Delta S+\Lambda(x)-S=0, & x \in \Omega \\ \frac{\partial S}{\partial \nu}=0, & x \in \partial \Omega .\end{cases}
$$

Similar to Theorem 3.5, we have

Theorem 6.1. If the basic reproduction number $\mathcal{R}_{0}<1$, then the unique globalin-time bounded and classical solution $(S, I)$ of the cross-diffusive SIS model (1.2) satisfies $(S, I) \rightarrow(\tilde{S}, 0)$ in $C(\bar{\Omega})$ as $t \rightarrow \infty$.

Proof. Let $v(x, t)=S(x, t)-\tilde{S}(x)+I(x, t)$. Then straightforward calculations from (1.2) show that $v$ satisfies

$$
\begin{cases}v_{t}=d_{S} \Delta v+\nabla \cdot\left(\left(d_{I}-d_{S}\right) \nabla I+\chi S \nabla I\right)-v+I, & x \in \Omega, t>0, \\ \frac{\partial v}{\partial \nu}=0, & x \in \partial \Omega, t>0, \\ v(x, 0)=S_{0}(x)-\tilde{S}(x)+I_{0}(x), & x \in \Omega .\end{cases}
$$

We multiply the first equation in (6.1) by $v$, integrate by parts and employ the Cauchy-Schwarz inequality to obtain

$$
\begin{aligned}
\frac{1}{2} \frac{d}{d t} \int_{\Omega} v^{2} d x= & -d_{S} \int_{\Omega}|\nabla v|^{2} d x-\left(d_{I}-d_{S}\right) \int_{\Omega} \nabla I \cdot \nabla v d x \\
& -\chi \int_{\Omega} S \nabla I \cdot \nabla v d x-\int_{\Omega} v^{2} d x+\int_{\Omega} I v d x \\
\leq & -d_{S} \int_{\Omega}|\nabla v|^{2} d x+\frac{d_{S}}{4} \int_{\Omega}|\nabla v|^{2} d x+\frac{\left(d_{I}-d_{S}\right)^{2}}{d_{S}} \int_{\Omega}|\nabla I|^{2} d x \\
& +\frac{d_{S}}{4} \int_{\Omega}|\nabla v|^{2} d x+\frac{\chi^{2}}{d_{S}} \int_{\Omega} S^{2}|\nabla I|^{2} d x-\int_{\Omega} v^{2} d x+\frac{1}{2} \int_{\Omega} v^{2} d x+\frac{1}{2} \int_{\Omega} I^{2} d x \\
\leq & -\frac{d_{S}}{2} \int_{\Omega}|\nabla v|^{2} d x+\left[\frac{\left(d_{I}-d_{S}\right)^{2}}{d_{S}}+\frac{\chi^{2}\|S(\cdot, t)\|_{L^{\infty}(\Omega)}^{2}}{d_{S}}\right] \int_{\Omega}|\nabla I|^{2} d x+\frac{1}{2} \int_{\Omega} I^{2} d x .
\end{aligned}
$$


The fact that $\mathcal{R}_{0}<1$ shows that (3.4) and (3.7) remain valid. Then upon an integration in the time variable and in view of (3.4) and (3.7), we find that

$$
\int_{0}^{\infty} \int_{\Omega}|\nabla v|^{2} d x d t<\infty
$$

Next, we shall prove a weak stabilization of $v_{t}$, an analog of Lemma 3.4. To this purpose, we first integrate by parts both sides of the PDE in (6.1), we end up with

$$
\frac{d}{d t} \int_{\Omega} v(x, t) d x=-\int_{\Omega} v(x, t) d x+\int_{\Omega} I(x, t) d x .
$$

Thanks to the exponential decay of $I$ in (3.4), we can easily infer from (6.3) that $\int_{\Omega} v(\cdot, t)$ decays exponentially to zero, and hence, its average $\bar{v}(t):=\frac{1}{|\Omega|} \int_{\Omega} v(\cdot, t)$ decays also exponentially to zero as $t \rightarrow \infty$. According to the Poincaré inequality, there exists some generic positive constant $C$ such that

$$
\|v(\cdot, t)\|_{L^{2}(\Omega)} \leq\|v(\cdot, t)-\bar{v}(t)\|_{L^{2}(\Omega)}+\|\bar{v}(t)\|_{L^{2}(\Omega)} \leq C\|\nabla v(\cdot, t)\|_{L^{2}(\Omega)}+\|\bar{v}(t)\|_{L^{2}(\Omega)} .
$$

By the exponential decay of $\bar{v}(t)$ and (6.2), it then follows that

$$
\int_{0}^{\infty}\|v(\cdot, t)\|_{L^{2}(\Omega)}^{2} d t \leq 2 C^{2} \int_{0}^{\infty}\|\nabla v(\cdot, t)\|_{L^{2}(\Omega)}^{2} d t+2|\Omega| \int_{0}^{\infty}|\bar{v}(t)|^{2} d t<\infty .
$$

Now, for any test function $\varphi \in H^{1}(\Omega)$, we deduce from (6.1) that

$$
\begin{aligned}
\int_{\Omega} v_{t} \varphi d x= & -d_{S} \int_{\Omega} \nabla v \cdot \nabla \varphi d x-\int_{\Omega} v \varphi d x+\int_{\Omega} I \varphi d x \\
& -\int_{\Omega}\left[\left(d_{I}-d_{S}\right) \nabla I+\chi S \nabla I\right] \cdot \nabla \varphi d x \\
\leq & d_{S}\|\nabla v(\cdot, t)\|_{L^{2}(\Omega)}\|\nabla \varphi\|_{L^{2}(\Omega)}+\left(\|v(\cdot, t)\|_{L^{2}(\Omega)}+\|I(\cdot, t)\|_{L^{2}(\Omega)}\right)\|\varphi\|_{L^{2}(\Omega)} \\
& +\left[\left(d_{I}+d_{S}\right)\|\nabla I(\cdot, t)\|_{L^{2}(\Omega)}+\chi\|S(\cdot, t)\|_{L^{\infty}(\Omega)}\|\nabla I(\cdot, t)\|_{L^{2}(\Omega)}\right]\|\nabla \varphi\|_{L^{2}(\Omega)} \\
\leq & {\left[d_{S}\|\nabla v(\cdot, t)\|_{L^{2}(\Omega)}+\left(d_{I}+d_{S}+\chi\|S(\cdot, t)\|_{L^{\infty}(\Omega)}\right)\|\nabla I(\cdot, t)\|_{L^{2}(\Omega)}\right.} \\
& \left.+\|v(\cdot, t)\|_{L^{2}(\Omega)}+\|I(\cdot, t)\|_{L^{2}(\Omega)}\right]\|\varphi\|_{H^{1}(\Omega)},
\end{aligned}
$$

which gives

$$
\begin{aligned}
\left\|v_{t}(\cdot, t)\right\|_{\left(H^{1}(\Omega)\right)^{*}} \leq & d_{S}\|\nabla v(\cdot, t)\|_{L^{2}(\Omega)}+\left(d_{I}+d_{S}+\chi\|S(\cdot, t)\|_{L^{\infty}(\Omega)}\right)\|\nabla I(\cdot, t)\|_{L^{2}(\Omega)} \\
& +\|v(\cdot, t)\|_{L^{2}(\Omega)}+\|I(\cdot, t)\|_{L^{2}(\Omega)} .
\end{aligned}
$$

Combining this with (3.4), (3.7), (6.2) and (6.4), we derive that

$$
\int_{0}^{\infty}\left\|v_{t}(\cdot, t)\right\|_{\left(H^{1}(\Omega)\right)^{*}}^{2} d t<\infty .
$$

With the help of (6.2) and (6.5), combined with the exponential decay of $\int_{\Omega} v(\cdot, t)$, using an argument similar to that of Theorem 3.5 , one can readily show that in fact $v(\cdot, t) \rightarrow 0$ in $C(\bar{\Omega})$ as $t \rightarrow \infty$. Since we have already known $I(\cdot, t) \rightarrow 0$ uniformly, then it follows $S(\cdot, t)=v(\cdot, t)+\tilde{S}-I(\cdot, t) \rightarrow \tilde{S}$ uniformly on $\bar{\Omega}$ as $t \rightarrow \infty$.

In the case $\mathcal{R}_{0}>1$, we have

Theorem 6.2. Suppose that all of $\beta, \gamma$ and $\Lambda$ are all positive constants and that $\beta>\gamma$ (so that $\mathcal{R}_{0}=\beta / \gamma>1$ ). Then there exists a positive constant $M_{1}$ depending only on $n, \Omega, \beta, \gamma, d_{I}$ and $\hat{N}$ such that whenever $0 \leq \chi<\chi_{0}:=M_{1} \sqrt{d_{S}}$, the unique 
classical global-in-time solution $(S, I)$ of (1.2) converges uniformly to the unique EE $(\hat{S}, \hat{I})$ in the following fashion:

$$
\lim _{t \rightarrow \infty}\left(\|S(\cdot, t)-\hat{S}\|_{L^{\infty}(\Omega)}+\|I(\cdot, t)-\hat{I}\|_{L^{\infty}(\Omega)}\right)=0
$$

where

$$
(\hat{S}, \hat{I})=\left(\Lambda, \frac{\beta-\gamma}{\gamma} \Lambda\right)
$$

Proof. The proof is very similar to that of Theorem 3.6 (i) by using the same Lyapunov functional; the details are thus omitted here.

In the general situation, as in proving Theorem 3.7 for the system (1.1), we can employ the abstract dynamical systems theory to conclude the uniform persistence property for the system (1.2). That is, we can state

Theorem 6.3. Let $\left(u_{0}, v_{0}\right)$ obey (1.3) and $\mathcal{R}_{0}>1$. Then system (1.2) is uniformly persistent, i.e., there exists some $\eta>0$, independent of $\left(u_{0}, v_{0}\right)$, such that

$$
\liminf _{t \rightarrow \infty} S(x, t) \geq \eta \text { and } \liminf _{t \rightarrow \infty} I(x, t) \geq \eta \text { uniformly for } x \in \bar{\Omega} .
$$

Furthermore, (1.2) admits at least an $E E(S, I)$.

Hence, when $\mathcal{R}_{0}>1$, Theorem 6.3 ensures the existence of EE to (1.2), which solves the following elliptic problem:

$$
\begin{cases}d_{S} \Delta S+\chi \nabla \cdot(S \nabla I)-\beta(x) \frac{S I}{S+I}+\gamma I+\Lambda(x)-S=0, & x \in \Omega, \\ d_{I} \Delta I+\beta(x) \frac{S I}{S+I}-\gamma(x) I=0, & x \in \Omega, \\ \frac{\partial S}{\partial \nu}=\frac{\partial I}{\nu}=0, & x \in \partial \Omega .\end{cases}
$$

For (6.6), we only capture the following information about the asymptotic profile of $\mathrm{EE}$ of (1.2) as $d_{S} \rightarrow 0$, which is poorer than that of (4.1).

Theorem 6.4. Assume that $\mathcal{R}_{0}>1$. Fix $d_{I}>0$ and let $d_{S} \rightarrow 0$, then every positive solution $(S, I)$ of the problem (6.6) satisfies (up to a subsequence of $d_{S} \rightarrow 0$ )

$$
I \rightarrow I^{*} \text { in } C^{1}(\bar{\Omega})
$$

for some positive function $I^{*} \in C^{1}(\bar{\Omega})$.

Proof. We integrate by parts from (6.6) to obtain

$$
-\int_{\Omega} \beta \frac{S I}{S+I} d x+\int_{\Omega} \gamma I d x+\int_{\Omega} \Lambda d x-\int_{\Omega} S d x=0,
$$

and

$$
\int_{\Omega} \beta \frac{S I}{S+I} d x-\int_{\Omega} \gamma I d x=0 .
$$

Adding the two identities, we see

$$
\int_{\Omega} S d x=\int_{\Omega} \Lambda d x
$$

Then (6.7) moreover gives

$$
\gamma_{*} \int_{\Omega} I d x \leq \int_{\Omega} \gamma I d x=\int_{\Omega} \beta \frac{S I}{S+I} d x \leq \beta^{*} \int_{\Omega} S d x=\beta^{*} \int_{\Omega} \Lambda d x .
$$


Thus, the $L^{1}$-norm of $S$ and $I$ is uniformly bounded with respect to $d_{S}>0$.

Now, thanks to (6.8), as in the proof of Theorem [5.2, after passing to a subsequence of $d_{S} \rightarrow 0$, it holds

$$
I \rightarrow I^{*} \geq 0 \text { in } C^{1}(\bar{\Omega}), \quad \text { as } d_{S} \rightarrow 0,
$$

for some $I^{*} \in C^{1}(\bar{\Omega})$. Furthermore, we have the dichotomy:

$$
I^{*}>0 \text { on } \bar{\Omega} \text { or } I^{*} \equiv 0 .
$$

Assume that $I^{*} \equiv 0$; that is, $I \rightarrow 0$ in $C^{1}(\bar{\Omega})$ as $d_{S} \rightarrow 0$. Expanding out the crossdiffusive term in the first equation of (6.6) and using the second equation, we see that $S$ satisfies

$$
\begin{cases}d_{S} \Delta S+\chi \nabla I \cdot \nabla S+\left(\gamma-\frac{\beta S}{S+I}\right)\left(1+\frac{\chi S}{d_{I}}\right) I+\Lambda-S=0, & x \in \Omega \\ \frac{\partial S}{\partial \nu}=0, & x \in \partial \Omega .\end{cases}
$$

Then, since $I \rightarrow 0$ in $C^{1}(\bar{\Omega})$ as $d_{S} \rightarrow 0$, as in the proof of (5.8), a singular perturbation argument applied to (6.9) yields that

$$
S \rightarrow \Lambda \quad \text { uniformly on } \bar{\Omega}, \quad \text { as } d_{S} \rightarrow 0 .
$$

Now, to proceed, we let

$$
h=\frac{I}{\|I\|_{L^{\infty}(\Omega)}}>0 .
$$

Then $\|h\|_{L^{\infty}(\Omega)}=1$ and it can be easily seen that $h$ satisfies

$$
\begin{cases}d_{I} \Delta h+\left(\beta \frac{S}{S+I}-\gamma\right) h=0, & x \in \Omega \\ \frac{\partial h}{\partial \nu}=0, & x \in \partial \Omega .\end{cases}
$$

The Harnack inequality [28, Lemma 2.2] tells us that

$$
\max _{\bar{\Omega}} h \leq C \min _{\bar{\Omega}} h
$$

for some positive constant $C$ independent of $d_{S}>0$. Since $h$ is uniformly bounded by 1 , after passing to a subsequence of $d_{S} \rightarrow 0$, we have, as before,

$$
h \rightarrow \tilde{h} \text { in } C^{1}(\bar{\Omega}),
$$

for some $0 \leq \tilde{h} \in C^{1}(\bar{\Omega})$ with $\|\tilde{h}\|_{L^{\infty}(\Omega)}=1$. In light of the Harnack inequality (6.11), it is necessary that $\tilde{h}>0$ on $\bar{\Omega}$. On the other hand, since $S \rightarrow \Lambda>0$ and $I \rightarrow 0$ uniformly on $\bar{\Omega}$ as $d_{S} \rightarrow 0$, it follows from (6.10) that $\tilde{h}$ satisfies

$$
\begin{cases}d_{I} \Delta \tilde{h}+(\beta-\gamma) \tilde{h}=0, & x \in \Omega, \\ \frac{\partial \tilde{h}}{\partial \nu}=0, & x \in \partial \Omega .\end{cases}
$$

This says that the principal eigenvalue $\lambda^{*}$ of the eigenvalue problem (3.2) is zero, contradicting $\mathcal{R}_{0}>1$ and the fact that $1-\mathcal{R}_{0}$ and $\lambda^{*}$ have the same sign. Therefore, it holds

$$
I \rightarrow I^{*}>0 \text { in } C^{1}(\bar{\Omega}), \quad \text { as } d_{S} \rightarrow 0 .
$$

This finishes the proof. 
Acknowledgments. We would like to thank the two anonymous referees for their careful reading of our manuscript, and constructive comments and suggestions, which further help us to improve the presentation.

\section{REFERENCES}

[1] N. Alikakos, An application of the invariance principle to reaction-diffusion equations, J. Differential Equations 33 (1979), 201-225.

[2] L.J.S. Allen, B.M. Bolker, Y. Lou, A.L. Nevai, Asymptotic profiles of the steady states for an SIS epidemic disease patch model, SIAM J. Appl. Math. 67(2007), 1283-1309.

[3] L.J.S. Allen, B.M. Bolker, Y. Lou, A.L. Nevai, Asymptotic profiles of the steady states for an SIS epidemic reaction-diffusion model, Discrete Contin. Dyn. Syst. 21 (2008), 1-20.

[4] N. Bellomo, A. Bellouquid, Y. Tao, M. Winkler, Toward a mathematical theory of Keller-Segel models of pattern formation in biological tissues, Math. Models Methods Appl. Sci. 25 (2015), 1663-1763.

[5] K.J. Brown, P.C. Dunne, R.A. Gardner, A semilinear parabolic system arising in the theory of superconductivity, J. Differential Equations 40 (1981), 232-252.

[6] X. Cao, Global bounded solutions of the higher-dimensional Keller-Segel system under smallness conditions in optimal spaces, Discrete Contin. Dyn. Syst. 35 (2015), 1891-1904.

[7] T. Cieślak, Ph. Laurençt and C. Morales-Rodrigo, Global existence and convergence to steady states in a chemorepulsion system, equations, in Parabolic and Navier-Stokes Equations, in: Banach Center Publ. Polish Acad. Sci. Inst. Math., 81 (2008), 105-117.

[8] J. Cui, X. Tao, H. Zhu, An SIS infection model incorporating media coverage, Rocky Mount. J. Math. 38 (2008), 1323-1334.

[9] R. Cui, K.-Y. Lam, Y. Lou, Dynamics and asymptotic profiles of steady states of an epidemic model in advective environments, J. Differential Equations, 263 (2017), 2343-2373.

[10] R. Cui, Y. Lou, A spatial SIS model in advective heterogeneous environments, J. Differential Equations, 261 (2016), 3305-3343.

[11] K. Deng, Y. Wu, Dynamics of a susceptible-infected-susceptible epidemic reaction-diffusion model, Proc. Roy. Soc. Edinburgh Sect. A 146 (2016), 929-946.

[12] W. Ding, W. Huang, S. Kansakar, Traveling wave solutions for a diffusive SIS epidemic model, Discrete Contin. Dyn. Syst. Ser. B 18 (2013), 1291-1304.

[13] Y. Du, R. Peng, M. Wang, Effect of a protection zone in the diffusive Leslie predator-prey model, J. Differential Equations 246 (2009), 3932-3956.

[14] A. Friedman, Partial differential equations of parabolic type, Prentice-Hall, Inc., Englewood Cliffs, N.J. 1964 xiv +347 pp.

[15] D. Gao, S. Ruan, An SIS patch model with variable transmission coefficients, Math. Biosci. 232 (2011), 110-115.

[16] J. Ge, K.I. Kim, Z. Lin, H. Zhu, A SIS reaction-diffusion-advection model in a low-risk and high-risk domain, J. Differential Equations, 259 (2015), 5486-5509.

[17] D. Horstmann, M. Winkler, Boundedness vs. blow-up in a chemotaxis system, J. Differential Equations 215 (2005), 52-107.

[18] W. Huang, M. Han, K. Liu, Dynamics of an SIS reaction-diffusion epidemic model for disease transmission, Math. Biosci. Eng. 7 (2010), 51-66.

[19] V. Hutson, Y. Lou, K. Mischaikow, Convergence in competition models with small diffusion coefficients, J. Differffential Equations 211 (2005), 135-161.

[20] W. Jäger, S. Luckhaus, On explosions of solutions to a system of partial differential equations modelling chemotaxis, Trans. Amer. Math. Soc. 329 (1992), 819-824.

[21] H.-Y. Jin, T. Xiang, Boundedness and exponential convergence in a chemotaxis model for tumor invasion, Nonlinearity 29 (2016), 3579-3596.

[22] K. Kuto, H. Matsuzawa, R. Peng, Concentration profile of the endemic equilibria of a reactiondiffusion-advection SIS epidemic model, Calc. Var. Partial Differential Equations, to appear.

[23] O. Ladyzhenskaya, V. Solonnikov, N. Uralceva, Linear and Quasilinear Equations of Parabolic Type, AMS, Providence, RI, 1968.

[24] H. Li, R. Peng, F.-B. Wang, Varying total population enhances disease persistence: Qualitative analysis on a diffusive SIS epidemic model, J. Differential Equations, 262 (2017), 885-913. 
[25] T. Li, R. Pan, K. Zhao, Global dynamics of a hyperbolic-parabolic model arising from chemotaxis, SIAM J. Appl. Math., 72 (2012), 417-443.

[26] Y. Lou, W.-M. Ni, Diffusion, self-diffusion and cross-diffusion, J. Differential Equation 131 (1996), 79-131.

[27] P. Magal, X.-Q. Zhao, Global attractors and steady states for uniformly persistent dynamical systems, SIAM. J. Math. Anal. 37 (2005), 251-275.

[28] R. Peng, Asymptotic profiles of the positive steady state for an SIS epidemic reaction-diffusion model. I, J. Differential Equations 247 (2009), 1096-1119.

[29] R. Peng, S. Liu, Global stability of the steady states of an SIS epidemic reaction-diffusion model, Nonlinear Anal. 71 (2009), 239-247.

[30] R. Peng, F. Yi, Asymptotic profile of the positive steady state for an SIS epidemic reactiondiffusion model: effects of epidemic risk and population movement, Phys. D 259 (2013), 8-25.

[31] R. Peng, X.-Q. Zhao, A reaction-diffusion SIS epidemic model in a time-periodic environment, Nonlinearity 25 (2012), 1451-1471.

[32] M.M. Porzio and V. Vespri, Hölder estimates for local solutions of some doubly nonlinear degenerate parabolic equations, J. Differential Equations, 103 (1993), 146-178.

[33] Y. Tao, Global dynamics in a higher-dimensional repulsion chemotaxis model with nonlinear sensitivity, Discrete Contin. Dyn. Syst. Ser. B, 18 (2013), 2705-2722.

[34] Y. Tao, Z.-A. Wang, Competing effects of attraction vs. repulsion in chemotaxis, Math. Models Methods Appl. Sci., 23 (2013), 1-36.

[35] Y. Tao, M. Winkler, Eventual smoothness and stabilization of large-data solutions in a threedimensional chemotaxis system with consumption of chemoattractant, J. Differential Equations 252 (2012) 2520-2543.

[36] M. Winkler, Aggregation vs. global diffusive behavior in the higher-dimensional Keller-Segel model, J. Differential Equations 248 (2010), 2889-2905.

[37] M. Winkler, Boundedness in the higher-dimensional parabolic-parabolic chemotaxis system with logistic source, Comm. Partial Differential Equations 35 (2010), 1516-1537.

[38] M. Winkler, Blow-up in a higher-dimensional chemotaxis system despite logistic growth restriction, J. Math. Anal. Appl. 384 (2011), 261-272.

[39] M. Winkler, Finite-time blow-up in the higher-dimensional parabolic-parabolic Keller-Segel system, J. Math. Pures Appl. 100 (2013), 748-767.

[40] M. Winkler, Stabilization in a two-dimensional chemotaxis-Navier-Stokes system, Arch. Ration. Mech. Anal. 211 (2014), 455-487.

[41] Y. Wu, X. Zou, Asymptotic profiles of steady states for a diffusive SIS epidemic model with mass action infection mechanism, J. Differential Equations, 261 (2016), 4424-4447.

[42] X. Zhao, Dynamical Systems in Population Biology, Second edition, CMS Books in Mathematics, Springer, Cham, 2017.

School of Mathematics, Sun Yat-sen University, Guangzhou, 510275, Guangdong Province, China

E-mail address: lihuicong@mail.sysu.edu.cn

School of Mathematics and Statistics, Jiangsu Normal University, Xuzhou, 221116, Jiangsu Province, China

E-mail address: pengrui_seu@163.com

Institute for Mathematical Sciences, Renmin University of China, Bejing, 100872, CHINA

E-mail address: txiang@ruc.edu.cn 